\title{
THE FINITE DISPLACEMENT OF THIN RODS $\left({ }^{1}\right)$
}

\author{
BY
}

G. E. HAY

Contents

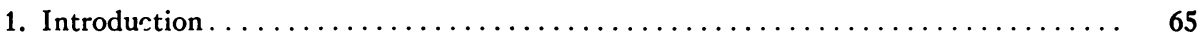

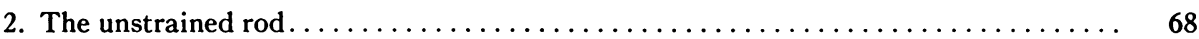

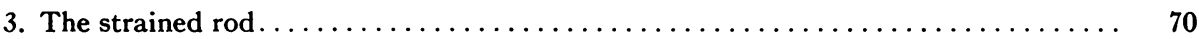

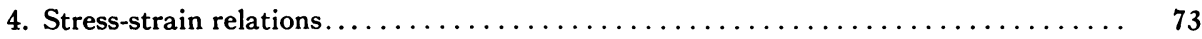

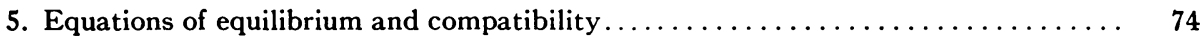

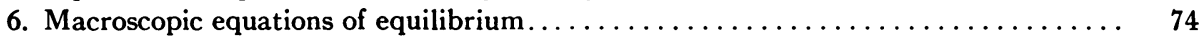

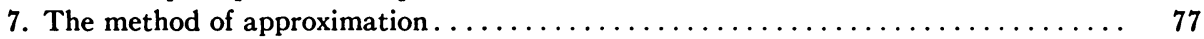

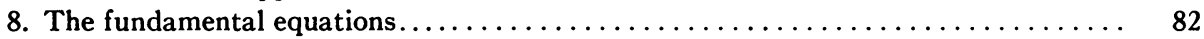

9. The first approximation: the stress $\ldots \ldots \ldots \ldots \ldots \ldots \ldots \ldots \ldots \ldots \ldots \ldots \ldots \ldots \ldots$

10. The first approximation: the macroscopic equations of equilibrium $\ldots \ldots \ldots 7$

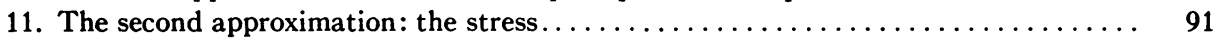

12. The second approximation: the macroscopic equations of equilibrium . . . . . .

13. The straightening of naturally curved thin rods by terminal force-systems . . . . . 94

14. Remarks on the experimental determination of elastic constants ............99

Appendix A................................... 100

1. Introduction. With reference to problems in elasticity, the terms "small displacement," "finite displacement," "small strain" and "finite strain" have the following meanings which will be made more precise later. If the displacement of every particle in a strained elastic body is much smaller than the greatest linear dimension of the body, the displacement is small; otherwise it is finite. If the elongation per unit length of every element in the body is much smaller than unity the strain is small; otherwise it is finite.

Only those problems involving small strain lie within the scope of ordinary mathematical elasticity. In most problems the strain is small only if the displacement is also small. However, in the case of thin rods and plates the displacement may be finite and yet the resulting strain may be small. Such finite displacement of thin rods will be considered in this paper. At a later date it is proposed to deal with the corresponding problem for thin plates.

The usual theory of the finite displacement of thin rods is due chiefly to Kirchhoff: it has been the subject of a number of papers going back nearly a hundred years $\left({ }^{2}\right)$. An account of the essentials of the Kirchhoff theory has

Presented to the Society, April 11, 1941; received by the editors December 4, 1940.

(1) This paper was written originally with the aid of a scholarship from the National Research Council of Canada, and was revised at Armour Institute of Technology, Chicago.

(2) B. de Saint-Venant, Comptes Rendus de l'Académie des Sciences, Paris, vol. 17 (1843), pp. 942-945, 1020-1031; vol. 19 (1844), pp. 36-44, 181-187. J. Binet, ibid., vol. 18 (1844), pp. 1115-1119. Wantzel, ibid., vol. 18 (1844), pp. 1197-1201. G. Kirchhoff, Journal für die reine und angewandte Mathematik (Crelle), vol. 56 (1859), pp. 285-313, or Vorlesungen über 
been given by Love $\left(^{3}\right)$. This theory appears to be in need of certain improvements which it is the purpose of the present paper to present. First, in order to deduce the equations governing the finite displacement of a thin rod naturally curved, it was necessary to assume that the rod was displaced to its final position through an intermediate stage in which the rod was straight. No such artificiality is required in the present paper. Secondly, approximation was introduced in a somewhat haphazard fashion. In the present paper approximation is introduced systematically in a manner which permits a theoretical solution of the problem to any desired degree of accuracy. The method is that of development in a power series in a small dimensionless parameter $\epsilon$ which tends to zero with the sectional area of the rod. This method was used by G. D. Birkhoff and others for thin plates $\left({ }^{4}\right)$; it has also been used for thin membranes $\left({ }^{5}\right)$ and for beams undergoing small displacement $\left(^{6}\right)$.

Synopsis. In this paper we consider rods of uniform cross-section with the external forces acting only on the ends.

The method of tensor calculus is used. Solutions are sought for the equations of equilibrium and compatibility by means of a systematic method of approximation. These solutions contain certain arbitrary functions which can be determined by applying the same method of approximation to certain macroscopic equations of equilibrium. These macroscopic equations of equilibrium express the fact that each element of the rod lying between adjacent cross-sections is in equilibrium under the action of forces acting on its ends. Even though the strain is small, in this work we require stress-strain relations which are more accurate than the usual relations. These relations involve five elastic constants whereas the usual relations involve only two.

The method of approximation hinges on a dimensionless parameter $\epsilon$ introduced in the following manner. We consider a singly infinite sequence of thin rods with identical lines of centroids of equal lengths, and with cross-

mathematische Physik, Mechanik, 3rd edition, Leipzig, 1883. A. Clebsch, Theorie der Elasticität, Leipzig, 1862, Part 4, or Théorie de l'Elasticité, Paris, 1881, chap. 4. A. B. Basset, Proceedings of the London Mathematical Society, vol. 23 (1891-1892), pp. 105-127; American Journal of Mathematics, vol. 17 (1895), pp. 281-317.

(3) A. E. H. Love, Elasticity, 4th edition, Cambridge, 1934, chap. 18.

(4) G. D. Birkhoff, Philosophical Magazine, vol. 43 (1922), pp. 953-962. C. A. Garabedian, these Transactions, vol. 25 (1923), pp. 343-398; Comptes Rendus de l'Académie des Sciences, Paris, vol. 177 (1923), pp. 942-944; vol. 178 (1924), pp. 619-621; vol. 179 (1924), pp. 381-384; vol. 180 (1925), pp. 257-259; vol. 181 (1925), pp. 319-321; vol. 186 (1928), pp. 1518-1520; vol. 195 (1932), pp. 1369-1371. H. W. Sibert, these Transactions, vol. 33 (1931), pp. 329-369. R. Higdon and D. L. Holl, Duke Mathematical Journal, vol. 3 (1937), pp. 18-34.

${ }^{(5)}$ J. L. Synge, Philosophical Transactions of the Royal Society, (A), vol. 231 (1933), pp. 435-477; Transactions of the Royal Society of Canada, (3), section III, vol. 31 (1937), pp. 57-81. G. E. Hay, Canadian Journal of Research, (A), vol. 17 (1939), pp. 106-121; vol. 17 (1939), pp. 123-1i0.

$\left(^{6}\right)$ J. N. Goodier, Transactions of the Royal Society of Canada, (3), section III, vol. 32 (1938), pp. 1-25. 
sections having average diameters continuously decreasing to zero. We define $\epsilon$ by the relation

$$
\epsilon=\frac{\text { average diameter of cross-section of unstrained rod }}{\text { length of unstrained rod }}
$$

$\epsilon$ then varies in the interval $0<\epsilon<\delta$, where $\delta$ is much smaller than unity.

Frequently we shall find it convenient to refer to the order of magnitude of certain quantities. By definition, a quantity $q$ is said to have the order of magnitude of $\epsilon^{r}$, or to be of order $\epsilon^{r}$, if $q / \epsilon^{r}$ tends to a finite nonzero limit as $\epsilon$ tends to zero. Quantities of order $\epsilon^{0}$ are said to be finite.

Let us now consider the problem as a whole. A rod of given geometrical form is subjected at one end to a given force-system (force and couple). It takes up some equilibrium configuration under the action of the given forcesystem and a suitable force-system applied to the other end. Thus the problem may be considered as characterized by

(a) the geometrical form of the unstrained rod,

(b) the force-system applied to one end.

When these are given, the problem is to determine the geometrical form of the unstrained rod by solving the equations of equilibrium and compatibility together with the macroscopic equations of equilibrium. It is found that-

(a) When the applied force-system is of order $\epsilon^{2}$ or lower, the strain is finite.

(b) When the applied force-system is of order $\epsilon^{5}$ or higher the displacement is small.

(c) When the applied force-system is of order $\epsilon^{3}$ or $\epsilon^{4}$, the displacement is in general finite, while the strain is either finite or small depending on the configuration of the unstrained rod and on the individual components of the applied force-system. We are interested only in those problems in which the displacement is finite and the strain small. It is found that all problems in which this is the case fall naturally into three classes which will now be discussed in turn.

In problems of Class (i) the unstrained rod has in general an arbitrary finite twist and curvature. The given force-system applied to one end is of order $\epsilon^{4}$ but is otherwise arbitrary. In the strained rod the twist and curvature are in general finite and can be found by the first approximation (Kirchhoff theory) while the elongation per unit length is of order $\epsilon^{2}$ and can be found only by recourse to the second approximation.

In problems of Class (ii) the unstrained rod has again in general an arbitrary finite twist and curvature, but the given force-system applied to one end of the rod consists of an arbitrary force of order $\epsilon^{3}$ (much larger than in Class (i)), an arbitrary twisting couple of order $\epsilon^{4}$, and a particular bending couple of order $\epsilon^{4}$. The strained rod is nearly straight, with its line of centroids coinciding approximately with the line of action of the given force applied 
to the end. In the strained rod the twist (which is finite), the curvature (of order $\epsilon$ ) and the elongation per unit length (of order $\epsilon$ ) can all be found by the first approximation. When the magnitude of the curvature is reduced in this way from order $\epsilon^{0}$ (finite) to order $\epsilon$ (small), the rod will be referred to as "straightened." An example of such straightening is worked out in $\$ 13$.

Class (iii) is a particular case of Class (i). It arises when the form of the unstrained rod is such that two special conditions ((10.20) or (10.21)) are satisfied and the given terminal force-system consists of an arbitrary force and a particular bending couple both of order $\epsilon^{4}$, the bending couple being so chosen that the strained rod is "straightened." In the strained rod the twist is the same as in the unstrained state, while the curvature (of order $\epsilon$ ) and the elongation per unit length (of order $\epsilon^{2}$ ) can be found only by recourse to the second approximation. A problem of Class (iii) is worked out in $\$ 13$.

2. The unstrained rod. A mathematical investigation involving rods is greatly facilitated by the use of such concepts as "normal cross-section" and "line of centroids." Since these are rather difficult to define for a general curved rod, the present investigation will be limited to those rods which, in the unstrained state, can be defined in the following manner. In Figure 1a,

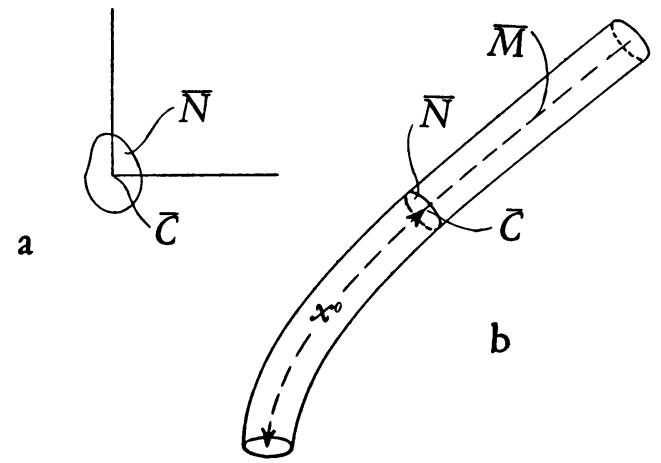

FIg. 1. The unstrained rod: $\bar{N}$ is a cross-section of the rod, $\bar{C}$ is the centroid of $\bar{N}$, and $\bar{M}$ is the line of centroids

$\bar{N}$ is a region bounded by a general closed plane curve, and $\bar{C}$ is the centroid of $\bar{N}$. In Figure $1 \mathrm{~b}, \bar{M}$ is a general regular skew curve. The rod is generated by $\bar{N}$ when $\bar{C}$ moves along $\bar{M}$ with the plane of $\bar{N}$ normal to $\bar{M}$, as shown in Figure $1 \mathrm{~b}$. When a rod is defined in this manner, it has a uniform normal crosssection $\bar{N}$ and a line of centroids $\bar{M}$. Since the rod is to be thin, it is certainly necessary that

(maximum diameter of $\bar{N}) /($ length of $\bar{M})<1$.

Curvilinear coordinates $x^{i}(i=0,1,2)$ will be used: $x^{0}$ is the arc length measured along $\bar{M}$ from one end of the rod to $\bar{C}: x^{1}$ and $x^{2}$ are rectangular cartesian coordinates in the cross-section through $\bar{C}$, with origin at $\bar{C}$ and 
the principal axes of inertia of the cross-section as axes. Latin indices will have the range $0,1,2$; Greek indices the range 1,2; and, in accordance with the usual convention, repeated indices will indicate summation over the appropriate ranges. The line element in the rod will then be of the form

$$
d \bar{s}^{2}=\bar{g}_{i j} d x^{i} d x^{i},
$$

where $\bar{g}_{i j}$ is the metric tensor and $\bar{g}_{i j}=\bar{g}_{j i}$. Because of the nature of the coordinate system,

$$
\left(\bar{g}_{00}\right) \bar{c}=1, \quad\left(\bar{g}_{0 \alpha}\right) \bar{c}=0, \quad \bar{g}_{\alpha \beta}=\delta_{\beta}^{\alpha},
$$

where $\delta_{\beta}^{\alpha}$ is the Kronecker delta and $\left(\bar{g}_{i j}\right) \bar{c}$ means that $\bar{g}_{i j}$ is to be evaluated at the point $\bar{C}$ on $\bar{M}$ (where $x^{\alpha}=0$ ).

At each point $\bar{C}$ on $\bar{M}$ there is an orthogonal triad associated with the coordinate axes. To describe the configuration of $\bar{M}$, we shall use a rotation vector $\bar{\omega}^{i}$ specifying the rotation of this triad as $\bar{C}$ moves along $\bar{M}: \bar{\omega}^{i} \bar{\lambda}_{i}$ is then the rotation per unit length about the direction of a unit vector $\bar{\lambda}_{i}$, and $\bar{\omega}^{0}$ and $\bar{\omega}^{\alpha}$ will be referred to respectively as the twist and curvature of the unstrained rod. The use of $\bar{\omega}^{\alpha}$ rather than the ordinary components of curvature brings a greater symmetry into subsequent equations.

The remainder of this section is devoted to the derivation of $(2.8)$, which expresses $\bar{\omega}^{i}$ in terms of $\bar{g}_{i j}$.

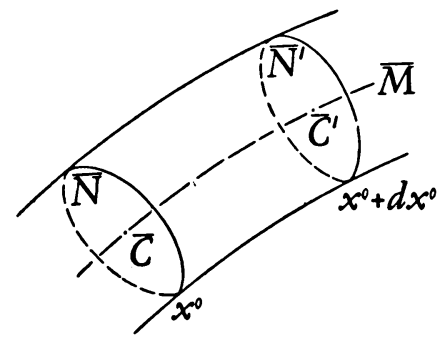

FIG. 2. A part of the unstrained rod

Two adjacent points $\bar{C}\left(x^{0}\right)$ and $\bar{C}^{\prime}\left(x^{0}+d x^{0}\right)$ are taken on the line of centroids $\bar{M}$ (Figure 2): $\bar{N}$ and $\bar{N}^{\prime}$ denote the cross-sections at $\bar{C}$ and $\bar{C}^{\prime}$, respectively. The positions relative to $\bar{C}$ of points lying in $\bar{N}$ and near $\bar{C}$ will be denoted by infinitesimal vectors $\left(\delta x^{\alpha}\right) \bar{c}$. Similarly the positions relative to $\bar{C}^{\prime}$ of points lying in $\bar{N}^{\prime}$ will be denoted by $\left(\delta x^{\alpha}\right) \bar{c}^{\prime}$. Corresponding to any point in $\bar{N}$ there is a point in $\bar{N}^{\prime}$ for which $\left(\delta x^{\alpha}\right) \bar{c}=\left(\delta x^{\alpha}\right) \bar{c}^{\prime}$, and all the points in $\bar{N}$ can be brought into coincidence with the corresponding points in $\bar{N}^{\prime}$ by a rigid-body displacement, which can be accomplished in two steps: (i) a rigidbody translation in which $\bar{C}$ moves into coincidence with $\bar{C}^{\prime}$ and $\bar{N}$ moves into a new position $\bar{N}^{\prime \prime}$; (ii) a rigid-body rotation in which each point in $\bar{N}^{\prime \prime}$ moves into coincidence with the corresponding point in $N^{\prime}$. 
In step (i), each vector $\left(\delta x^{\alpha}\right)_{\bar{C}}$ is propagated parallelly from $\bar{C}$ to $\bar{C}^{\prime}$ : The $x^{0}$-component of all vectors in the field produced by this parallel propagation vanishes in general only at $\bar{C}$. Presently, differentiation will be performed with respect to the components at $\bar{C}$ of this vector field. Thus it is necessary to introduce a third component $\left(\delta x^{0}\right)$ - which will be allowed to vanish after the differentiation has been performed. As a result of step (i), $\left(\delta x^{i}\right) \bar{c}$ becomes an infinitesimal vector in $\bar{N}^{\prime \prime}$ at $\bar{C}^{\prime}$ with components

$$
\left(\delta x^{i}\right)_{\bar{c}}-\left(\bar{F}_{j 0}^{i}\right)_{\bar{c}}\left(\delta x^{j}\right) \bar{c} d x^{0},
$$

where $\bar{F}_{j k}^{i}$ is the Christoffel symbol of the second kind in $\bar{g}_{i j}$,

$$
\bar{F}_{j k}^{i}=\frac{1}{2} b^{i l}\left(\frac{\partial \bar{g}_{k l}}{\partial x^{j}}+\frac{\partial \bar{g}_{l j}}{\partial x^{k}}-\frac{\partial \bar{g}_{j k}}{\partial x^{l}}\right):
$$

$b^{i j}$ is the minor of $\bar{g}_{i j}$ in the determinant $\left|\bar{g}_{i j}\right|$ divided by that determinant, and $\left(\bar{F}_{j k}^{i}\right) \bar{c}$ represents $\bar{F}_{j k}^{i}$ evaluated at $\bar{C}$ (where $x^{\alpha}=0$ ).

As a result of step (ii), (2.3) becomes $\left(\delta x^{i}\right) \bar{c}^{\prime}$, and since $\left(\delta x^{i}\right) \bar{c}=\left(\delta x^{i}\right) \bar{c}^{\prime}$, the infinitesimal displacement produced by the rigid-body rotation in step (ii) is

$$
\bar{u}^{i}=\left(\bar{F}_{j 0}^{i}\right) \bar{c}\left(\delta x^{j}\right) \bar{c} d x^{0} .
$$

This rigid-body rotation is specified by the skew-symmetric tensor

$$
\bar{\Omega}_{i j}=\frac{1}{2}\left(\frac{\partial \bar{u}_{j}}{\partial\left(\delta x^{i}\right)_{\bar{c}}^{-}}-\frac{\partial \bar{u}_{i}}{\partial\left(\delta x^{j}\right) \bar{c}}\right)=\frac{1}{2} d x^{0}\left(\frac{\partial \bar{g}_{0 j}}{\partial x^{i}}-\frac{\partial \bar{g}_{0 i}}{\partial x^{j}}\right) .
$$

It can also be specified by the contravariant vector $\bar{\omega}^{i} d x^{0}$, such that $\bar{\lambda}_{i} \bar{\omega}^{i} d x^{0}$ is the angle of rotation about a direction specified by a unit vector $\bar{\lambda}_{i}$. If $\bar{g}=\left|\bar{g}_{i j}\right|$ and $c^{i j k}$ are the permutation symbols $\left({ }^{7}\right)$, then

$$
\bar{\omega}^{i} d x^{0}=\frac{1}{2}\left(\bar{g}^{-1 / 2}\right) \bar{c} c^{i j k} \bar{\Omega}_{i k},
$$

whence, since $(\bar{g})_{C}=0$ by $(2.2)$,

$$
\bar{\omega}^{i}=\frac{1}{2} c^{i j k}\left(\frac{\partial \bar{g}_{0 k}}{\partial x^{j}}\right) . \bar{r} .
$$

The differentiation with respect to $\left(\delta x^{2}\right) \bar{c}$ has been performed and we now set $\left(\delta x^{0}\right)^{-}=0$. This does not alter (2.8), which thus expresses the rotation vector $\bar{\omega}^{i}$ of the unstrained rod in terms of $\bar{g}_{i j}$, as required.

3. The strained rod. When the rod defined in $\$ 2$ is acted upon by certain external forces, it is displaced finitely and experiences a small strain. The

( $\left.{ }^{7}\right)$ For an explanation of the permutation symbols and their tensor character, see, for example, O. Veblen, Invariants of Quadratic Differential Forms, Cambridge, 1927, pp. 3-4, 25-26. 
strained positions of the line of centroids $\bar{M}$, the cross-section $\bar{N}$, and the point $\bar{C}$ on both of them will be denoted by $M, N$ and $C$ respectively: $M, N$ and $C$ will be referred to as the strained line of centroids, the strained crosssection and the strained centroid of the cross-section of the rod, respectively. It will be noted that in general $N$ is a curved surface.

In the unstrained rod, the coordinates associated with a general particle are $x^{i}(i=0,1,2)$. In the strained rod the same coordinates will be used to specify the same particle. Such coordinates are called co-moving coordinates. They are a particular set belonging to the more general coordinates used by Murnaghan $\left.{ }^{8}\right)$. Thus, the line element in the strained rod will be of the form

$$
d s^{2}=g_{i j} d x^{i} d x^{j},
$$

where $g_{i j}$ is the metric tensor and $g_{i j}=g_{j i}$.

In $\$ 2$, a rotation vector $\bar{\omega}^{i}$ was determined for the unstrained rod. For the strained rod, a rotation vector $\omega^{i}$ can be defined somewhat analogously: $\omega^{0}$ and $\omega^{\alpha}$ will be referred to respectively as the twist and curvature of the strained rod. We shall now derive (3.7), which expresses $\omega^{i}$ in terms of $g_{i j}$ and is thus the analogue of $(2.8)$.

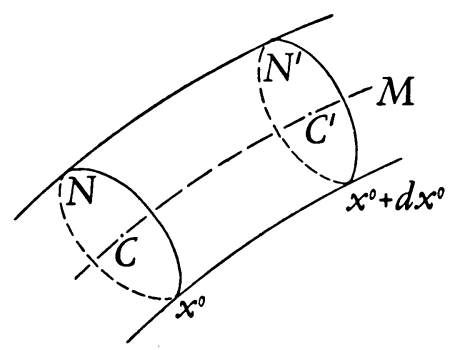

FIG. 3. A part of the strained rod

Two adjacent points $C\left(x^{0}\right)$ and $C^{\prime}\left(x^{0}+d x^{0}\right)$ are taken on the line of centroids $M$ (Figure 3 ): $N$ and $N^{\prime}$ denote the cross-sections at $C$ and $C^{\prime}$, respectively. The positions relative to $C$ of points lying in $N$ and near $C$ will be denoted by infinitesimal vectors $\left(\delta x^{\alpha}\right)_{c}$. Similarly the positions relative to $C^{\prime}$ of points lying in $N^{\prime}$ will be denoted by $\left(\delta x^{\alpha}\right)_{C^{\prime}}$. Corresponding to any point in $N$ and near $C$ there is a point in $N^{\prime}$ for which $\left(\delta x^{\alpha}\right)_{C}=\left(\delta x^{\alpha}\right)_{C^{\prime}}$, and each point in $N$ can be brought into coincidence with the corresponding point in $N^{\prime}$ by certain displacements. These displacements can be accomplished in two steps: (i) a rigid-body translation in which $C$ moves into coincidence with $C^{\prime}$ and $N$ moves into a new position $N^{\prime \prime}$; (ii) a system of displacements in which each point in $N^{\prime \prime}$ near $C^{\prime}$ moves into coincidence with the corresponding point in $N^{\prime}$.

In step (i), each vector $\left(\delta x^{\alpha}\right)_{C}$ is propagated parallelly from $C$ to $C^{\prime}$. Just

(8) F. D. Murnaghan, American Journal of Mathematics, vol. 59 (1937), pp. 235-260. 
as in $\S 2$, it is necessary to assume that the infinitesimal vectors $\left(\delta x^{\alpha}\right)_{c}$ have a third component $\left(\delta x^{0}\right)_{c}$ which can be allowed to vanish only after $\omega^{i}$ has been evaluated. Thus $\left(\delta x^{i}\right)_{c}$ becomes an infinitesimal vector in $N^{\prime \prime}$ at $C^{\prime}$ with components

$$
\delta x^{i}-\left(F_{j 0}^{i}\right)_{C}\left(\delta x^{j}\right)_{C} d x^{0},
$$

where $F_{j k}^{i}$ is the Christoffel symbol of the second kind in $g_{i j}$,

$$
F_{j k}^{i}=\frac{1}{2} g^{i l}\left(\frac{\partial g_{k l}}{\partial x^{j}}+\frac{\partial g_{l j}}{\partial x^{k}}-\frac{\partial g_{j k}}{\partial x^{l}}\right):
$$

$g^{i j}$ is the minor of $g_{i j}$ in the determinant $\left|g_{i j}\right|$ divided by that determinant, and $\left(F_{j k}^{i}\right)_{C}$ represents $F_{j k}^{i}$ evaluated at $C$ (where $x^{\alpha}=0$ ).

As a result of step (ii), (3.2) becomes $\left(\delta x^{i}\right)_{c^{\prime}}$; and since $\left(\delta x^{i}\right)_{C}=\left(\delta x^{i}\right)_{c^{\prime}}$, the system of infinitesimal displacements in step (ii) is

$$
u^{i}=\left(F_{j 0}^{i}\right)_{C}\left(\delta x^{j}\right)_{C} d x^{0} .
$$

This system of displacements can be considered as accomplished by means of a hypothetical homogeneous strain. This strain can be resolved into a pure strain and a rigid-body rotation. The rigid-body rotation is specified by the skew-symmetric tensor

$$
\Omega_{i j}=\frac{1}{2}\left(\frac{\partial u_{j}}{\partial\left(\delta x^{i}\right)_{C}}-\frac{\partial u_{i}}{\partial\left(\delta x^{j}\right)_{C}}\right)=\frac{1}{2} d x^{0}\left(\frac{\partial g_{0 j}}{\partial x^{2}}-\frac{\partial g_{0 i}}{\partial x^{j}}\right) .
$$

It can also be specified by a contravariant vector $\omega^{i} d x^{0}$ such that $\lambda_{i} \omega^{i} d x^{0}$ is the angle of rotation about a direction specified by a unit vector $\lambda_{i}$. If $g=\left|g_{i j}\right|$ and $c^{i j k}$ are the permutation symbols, then

$$
\omega^{i} d x^{0}=\frac{1}{2}\left(g^{-1 / 2}\right) c c^{i j k} \Omega_{j k},
$$

whence

$$
\omega^{i}=\frac{1}{2} c^{i j k}\left(g^{-1 / 2} \frac{\partial g_{0 k}}{\partial x^{i}}\right) .
$$

The differentiation with respect to $\left(\delta x^{i}\right)_{c}$ has been performed and we now set $\left(\delta x^{0}\right)_{c}=0$. This does not alter (3.7), which thus expresses the rotation vector $\omega^{i}$ of the strained rod in terms of $g_{i j}$, as required. This rotation vector refers to the rotation, as $C$ moves along $M$, of the principal axes at $C$ of the hypothetical strain introduced above. It will be noted that it does not refer to the rotation of a triad of vectors associated with the coordinate axes at $C$ as $C$ moves along the line of centroids $M$.

The elongation per unit length $e$ of an element in the rod is accurately

$$
e=\frac{d s-d \bar{s}}{d \bar{s}}=\left(g_{i j} d x^{i} d x^{j}\right)^{1 / 2}\left(\bar{g}_{k l} d x^{k} d x^{l}\right)^{-1 / 2}-1 .
$$


For the line of centroids,

$$
d x^{\alpha}=0,
$$

$\bar{g}_{i j}$ are as given in (2.2), and thus

$$
e=\left(g_{00}\right)_{c}^{1 / 2}-1,
$$

where the subscript $C$ means that the expression in parenthesis is to be evaluated at $C$ on the line of centroids.

It will be observed that all arguments in $\$ 3$ are purely geometrical, and do not actually involve any assumption that the strain is small.

4. Stress-strain relations. The strain tensor $\left({ }^{9}\right) e_{i j}$ is defined by

$$
2 e_{i j}=g_{i j}-\bar{g}_{i j} \text {. }
$$

Since the strain is to be small, the elongation per unit length of each element in the rod must be much smaller than unity. Thus, by (3.8), $e_{i j}$ must be $\ll 1$.

The usual stress-strain relations for an isotropic elastic body involve two elastic constants, and in their development all powers of the stress and strain tensors above the first are neglected. Stress-strain relations which involve five elastic constants, and in the development of which all powers of the stress and strain tensors above the second are neglected, have been deduced from considerations of elastic energy by Murnaghan $\left({ }^{10}\right)$. In this paper we shall require the latter relations, and in terms of a reduced stress tensor $T_{i j}$ defined by

$$
E_{i j}=E T_{i j}
$$

$E$ being Young's modulus and $E_{i j}$ the stress tensor, they take the form

$$
\begin{aligned}
e_{i j}= & -\sigma K_{1} g_{i j}+(1+\sigma) T_{i j}-\left(\sigma_{1}\left(K_{1}\right)^{2}+\sigma_{2} K_{2}\right) g_{i j} \\
& +\left(1+\sigma+\sigma_{2}\right) K_{1} T_{i j}+2(1+\sigma)^{2} T_{i k} g^{k l} T_{l j}+\sigma_{3} K_{i j},
\end{aligned}
$$

where $\sigma$ is Poisson's ratio, $\sigma_{1}, \sigma_{2}, \sigma_{3}$ are three new elastic constants which are dimensionless, and

$$
\begin{aligned}
K_{1} & =T_{\cdot i}^{i}, \\
2 K_{2} & =\delta_{k l}^{i j} T_{i}^{k} T_{j}^{l}, \\
2 K_{i j} & =g_{i p} \delta_{j m n}^{p k l} T_{k}^{m} T_{l}^{n},
\end{aligned}
$$

$\delta_{k,}^{u}, \delta_{j m n}^{p k l}$ being Kronecker deltas( $\left.{ }^{11}\right)$. If on the right side of (4.3) only the first two terms are retained, the two-constant stress-strain relations result.

(9) For a more detailed account of the application of tensor notation to elasticity, see, for example, P. Appell, Mecanique Rationnelle, vol. 5, Paris, 1926, p. 91.

$\left.{ }^{(10}\right)$ F. D. Murnaghan, loc. cit. See also L. Brillouin, Annales de Physique, vol. 3 (1925), pp. 251-297.

(11) For an explanation of the Kronecker deltas, see, for example, O. Veblen, loc. cit. 
5. Equations of equilibrium and compatibility. Except in \$2, which dealt with the geometry of the unstrained rod, throughout this paper the metric tensor $g_{i j}$ of the strained rod and its tensor reciprocal $g^{i j}$ will be used for lowering and raising suffixes, and also for forming tensorial derivatives.

Body forces are neglected. Thus, in terms of the reduced stress tensor $T_{i j}$, the equations of equilibrium are

$$
T^{i j}{ }_{j j}=0,
$$

where $\mid j$ signifies the covariant derivative. Since the space defined for the strained rod by (3.1) is flat, its curvature tensor $R_{i j k l}$ must vanish, i.e.,

$$
\begin{aligned}
2 R_{i j k l}= & \frac{\partial^{2} g_{i l}}{\partial x^{j} \partial x^{k}}+\frac{\partial^{2} g_{j k}}{\partial x^{i} \partial x^{l}}-\frac{\partial^{2} g_{i k}}{\partial x^{j} \partial x^{l}}-\frac{\partial^{2} g_{j l}}{\partial x^{i} \partial x^{k}} \\
& +2 g_{n p}\left(F_{j k}^{n} F_{i l}^{p}-F_{j l}^{n} F_{i k}^{p}\right)=0,
\end{aligned}
$$

where $F_{j k}^{t}$ are defined in (3.3). Contained in (5.2) are just six distinct equations,

$$
\begin{aligned}
& R_{0101}=R_{0102}=R_{0202}=0, \\
& R_{1220}=R_{1201}=R_{1212}=0 .
\end{aligned}
$$

In elastic problems involving small displacement only, (5.3) reduce to the usual equations of compatibility. Because of this, in the present work (5.3) will be called the equations of compatibility.

We let $n_{i}$ denote the covariant components of a unit vector pointing along the outward normal to the lateral surface of the rod. Thus, since no stress is transmitted across this surface,

$$
T_{i j} g^{j k} n_{k}=0 .
$$

6. Macroscopic equations of equilibrium. In this paper it is found necessary to introduce certain equations which express the equilibrium of each element of the rod lying between adjacent cross-sections. These equations will be called the macroscopic equations of equilibrium, the word "macroscopic" indicating that the equations involve an integration over the cross-section of the rod.

In Figure 3, $C$ is a point on the line of centroids $M$ of the strained rod and $N$ is the cross-section containing $C$. The reaction across $N$ is statically equivalent to a force at $C$ and a couple. The force will be denoted by $E P_{i}$ and the couple by $E Q_{i}$, where $E$ is Young's modulus. Thus, if $\left(\lambda^{i}\right)_{c}$ is any unit vector at $C$, then $E P_{i}\left(\lambda^{i}\right)_{C}$ and $E Q_{i}\left(\lambda^{i}\right)_{C}$ are, respectively, the component of the force in the direction $\left(\lambda^{i}\right)_{C}$ and the moment of the couple about that direction. The quantities $P_{i}$ and $Q_{i}$ are functions of $x^{0}$ only. They will be called reduced force and reduced couple, and are actually a force and a couple divided by Young's modulus. They satisfy the relations 


$$
\begin{aligned}
& P_{i}\left(\lambda^{i}\right)_{C}=\int_{N} T_{i j} \nu^{i} \lambda^{j} d S, \\
& Q_{i}\left(\lambda^{i}\right)_{C}=\int_{N} g^{-1 / 2} c^{i j k} \lambda_{i} s \mu_{j} T_{k l} \nu^{l} d S,
\end{aligned}
$$

where $d S$ is an element of area on $N, T_{i j}$ is the reduced stress tensor, $c^{i j k}$ is a permutation symbol, $g$ is the determinant $\left|g_{i j}\right|, \lambda^{i}$ is a unit vector defined at a point on $N$ by parallel propagation of $\left(\lambda^{i}\right)_{C}$ from $C, \nu^{i}$ is a unit vector normal to $N$ in the direction of increasing $x^{0}, \mu_{i}$ is a unit vector at $x^{\alpha}$ on $N$ tangent to the straight line drawn from $C$, and $s$ is the length of this straight line. The explicit evaluation of $P_{i}$ and $Q_{i}$ will be accomplished later $(\$ 8)$ by use of (6.1) and (6.2); and in preparation for this, expressions for $\lambda^{i}, \nu^{i}$ and $s \mu_{i}$ will now be derived in turn.

At points on $N$

$$
\lambda^{i}=\left(\lambda^{i}\right)_{c}+x^{\alpha}\left(\frac{\partial \lambda^{i}}{\partial x^{\alpha}}\right)_{C}+\cdots,
$$

and since $\lambda^{i}$ is propagated parallelly,

$$
\frac{\partial \lambda^{i}}{\partial x^{j}}+F_{j k}^{i} \lambda^{k}=0 .
$$

Thus

$$
\lambda^{i}=\left(\lambda^{i}\right)_{C}-x^{\alpha}\left(F_{\alpha k}^{i}\right)_{C}\left(\lambda^{k}\right)_{C}+\cdots
$$

For $\nu^{i}$ we have

$$
\nu_{0}=\left(g^{00}\right)^{-1 / 2}, \quad \nu_{\alpha}=0, \quad \nu^{0}=\left(g^{00}\right)^{1 / 2}, \quad \nu^{\alpha}=g^{\alpha 0}\left(g^{00}\right)^{-1 / 2} .
$$

The evaluation of $s \mu_{i}$ is more difficult. In order to give full power to the indicial notation, the coordinates of the initial point of the straight line of which $s$ is the length and $\mu_{i}$ the unit tangent vector at $x^{i}$ will be denoted by $a^{i}$. A straight line is a geodesic in three dimensions, and from variation of the end point of such a geodesic, it is known that

$$
\mu_{i}=\frac{\partial s}{\partial x^{i}}
$$

Also, for such a geodesic as long as the point $x^{i}$ is near the point $a^{i}$, it can be shown that

$$
\begin{aligned}
s^{2}= & \left(g_{i j}\right)_{x^{l}=a^{l}}\left(x^{i}-a^{i}\right)\left(x^{j}-a^{j}\right) \\
& +\left(g_{i j} F_{m n}^{j}\right)_{x^{l}=a l}\left(x^{i}-a^{i}\right)\left(x^{m}-a^{m}\right)\left(x^{n}-a^{n}\right)+\cdots,
\end{aligned}
$$

correct to the third order in $x^{i}-a^{i}$. Thus by (6.7), 


$$
\begin{aligned}
2 s \mu_{i}= & \frac{\partial s^{2}}{\partial x^{i}}=2\left(g_{i j}\right)_{x l=a l}\left(x^{j}-a^{j}\right) \\
& +\left(g_{i j} F_{m n}^{j}\right)_{x l=a l}\left(x^{m}-a^{m}\right)\left(x^{n}-a^{n}\right) \\
& +2\left(g_{m n} F_{i j}^{n}\right)_{x l=a l}\left(x^{m}-a^{m}\right)\left(x^{j}-a^{j}\right)+\cdots .
\end{aligned}
$$

In order to evaluate $s \mu_{i}$ as it occurs in (6.2), we set

obtaining

$$
a^{0}=x^{0}, \quad a^{\alpha}=0,
$$

$$
s \mu_{i}=\left(g_{i \alpha}\right)_{C} x^{\alpha}+\frac{1}{2}\left(g_{i j} F_{\alpha \beta}^{j}\right)_{C} x^{\alpha} x^{\beta}+\left(g_{\alpha n} F_{i \beta}^{n}\right)_{C} x^{\alpha} x^{\beta}+\cdots .
$$

In Figure 3, it is recalled that $C\left(x^{0}\right)$ and $C^{\prime}\left(x^{0}+d x^{0}\right)$ are adjacent points on the line of centroids $M$ of the strained rod, and that $N$ and $N^{\prime}$ are the crosssections containing $C$ and $C^{\prime}$, respectively. The conditions of statical equilibrium for the part of the rod lying between $N$ and $N^{\prime}$ are

$$
\begin{gathered}
-\left(\lambda^{i} P_{i}\right)_{C}+\left(\lambda^{i} P_{i}\right)_{C^{\prime}}=0, \\
-\left(\lambda^{i} Q_{i}\right)_{C}+\left(\lambda^{i} Q_{i}\right)_{C^{\prime}}+c^{i j k}\left(g^{-1 / 2} \lambda_{i} s \mu_{j} P_{k}\right)_{C^{\prime}}=0,
\end{gathered}
$$

where $P_{i}, Q_{i}$ and $g$ are as defined above, $\left(\lambda^{i}\right)_{C^{\prime}}$ is a unit vector at $C^{\prime}$ produced by parallel propagation of $\left(\lambda^{i}\right)_{C}$ from $C, \mu_{i}$ is the unit vector at $C^{\prime}$ tangent to the geodesic drawn from $C$ and $s$ is the length of this geodesic. Now

$$
\begin{aligned}
& \left(P_{i}\right)_{C^{\prime}}=\left(P_{i}\right)_{C}+\left(\frac{d P_{i}}{d x^{0}}\right)_{c} d x^{0}+\cdots, \\
& \left(Q_{i}\right)_{C^{\prime}}=\left(Q_{i}\right)_{C}+\left(\frac{d Q_{i}}{d x^{0}}\right)_{c} d x^{0}+\cdots, \\
& \left(\lambda^{i}\right)_{C^{\prime}}=\left(\lambda^{i}\right)_{c}+\left(\frac{\partial \lambda^{i}}{\partial x^{0}}\right)_{C} d x^{0}+\cdots
\end{aligned}
$$

Since $\lambda^{i}$ is propagated parallelly along $M,(6.14)$ can be written in the form

$$
\left(\lambda^{i}\right)_{C^{\prime}}=\left(\lambda^{i}\right)_{c}-\left(F_{j 0}^{i}\right)_{c}\left(\lambda^{j}\right)_{c} d x^{0}+\cdots,
$$

where $F_{j k}^{i}$ is the Christoffel symbol defined in (3.3). To evaluate $s \mu_{i}$ as it occurs in (6.12), we set $x^{\alpha}=a^{\alpha}=0, x^{0}-a^{0}=d x^{0}$ in (6.10). Then by substitution for $s \mu_{i}$, and for $\left(\lambda^{i}\right)_{c}$, from (6.15), it is found that (6.11) and (6.12) take the following forms, correct to the first order in $d x^{0}$,

$$
\begin{array}{r}
\left(\lambda^{i} \frac{d P_{i}}{d x^{0}}-F_{i 0}^{j} P_{j} \lambda^{i}\right)_{C}=0, \\
\left(\lambda^{i} \frac{d Q_{i}}{d x^{0}}-F_{i 0}^{j} Q_{j} \lambda^{i}+g^{-1 / 2} c^{j k l} g_{j 0} P_{k} g_{l i} \lambda^{i}\right)_{C}=0,
\end{array}
$$


whence, since $\left(\lambda^{i}\right)_{C}$ is an arbitrary unit vector,

$$
\begin{array}{r}
\frac{d P_{i}}{d x^{0}}-\left(F_{i 0}^{j}\right)_{C} P_{j}=0, \\
\frac{d Q_{i}}{d x^{0}}-\left(F_{i 0}^{j}\right)_{C} Q_{j}+\left(g^{-1 / 2} g_{i j} c^{j k l} g_{k 0}\right)_{C} P_{l}=0 .
\end{array}
$$

These are the macroscopic equations of equilibrium. They can be found easily in vector form by vector methods. However, in that form they do not lend themselves to the method of approximation used in the present paper.

7. The method of approximation. The metric tensor $g_{i j}$ of the strained rod, the strain tensor $e_{i j}$ and the reduced stress tensor $T_{i j}$ are all unknown. They are interrelated by (4.1) and by the stress-strain relations (4.3). They are to be determined by solving the equations of equilibrium (5.1) and the equations of compatibility (5.3). In order to solve these equations, approximation is introduced based on the thinness of the rod. In previous treatments of the problem, the manner in which this approximation was introduced suffered from certain defects which have been mentioned earlier. In this paper the approximation is introduced in the following systematic way.

We consider a singly infinite sequence of thin rods with identical lines of centroids of equal finite lengths, and similar cross-sections with average diameters continuously decreasing to zero. The relation

$$
\epsilon=\frac{\text { average diameter of cross-section of unstrained rod }}{\text { length of unstrained rod }}
$$

thus defines a dimensionless small parameter $\epsilon$ ranging continuously toward zero over values smaller than unity.

With regard to order of magnitude, a quantity $q$ will be referred to as of order $\epsilon^{r}(r=0,1,2, \cdots)$ if $q / \epsilon^{r}$ tends to a non-infinite, nonzero limit as $\epsilon$ tends to zero. A quantity of order $\epsilon^{0}$ will be referred to as finite. For example, if $l$ is the length of the line of centroids of the unstrained rod and $E$ is Young's modulus, then $l \epsilon^{r}$ is a length of order $\epsilon^{r}, E l^{2} \epsilon^{r}$ and $E l^{3} \epsilon^{r}$ are respectively a force and a couple of order $\epsilon^{r}, \epsilon^{r} / l$ is a curvature of order $\epsilon^{r}$, and so on for reduced force, reduced couple, etc.

We limit ourselves to rods of uniform cross-section, and since all rods in the sequence have similar cross-sections, the equation of their lateral surfaces takes the form

$$
f\left(\frac{x^{1}}{\epsilon}, \frac{x^{2}}{\epsilon}\right)=0 .
$$

It will be recalled that $x^{\alpha}(\alpha=1,2)$ are rectangular cartesian coordinates in a general normal cross-section of the unstrained rod, while $x^{0}$ is the arc-length 
of the line of centroids of the unstrained rod. Since the range of $x^{\alpha}$ is much smaller than that of $x^{0}$, new coordinates are introduced to replace $x^{\alpha}$. They are defined by

$$
x^{\alpha}=\epsilon \xi^{\alpha},
$$

so that the ranges of $x^{0}$ and $\xi^{\alpha}$ are all finite. Thus (7.2) becomes

$$
f\left(\xi^{1}, \xi^{2}\right)=0 .
$$

The metric tensor $\bar{g}_{i j}$ of the unstrained rod is assumed known. It is a function of $x^{0}$ and the relatively small coordinates $x^{\alpha}$. It is assumed that $\bar{g}_{i j}$ can be expanded in Taylor series in $x^{\alpha}$, and since

(by (2.2)), we then have

$$
\left(\bar{g}_{i j}\right)_{x \gamma_{=0}}=\delta_{j}
$$

$$
\bar{g}_{i j}=\delta_{j}^{i}+\epsilon \xi^{\alpha} \bar{g}_{i j}^{(\alpha)}+\frac{1}{2} \epsilon^{2} \xi^{\alpha} \xi^{\beta} \bar{g}_{i j}^{(\alpha \beta)}+\cdots,
$$

where

$$
\bar{g}_{i j}^{(\alpha)}=\left(\frac{\partial \bar{g}_{i j}}{\partial x^{\alpha}}\right)_{x^{\gamma}=0}, \quad \bar{g}_{i j}^{(\alpha \beta)}=\left(\frac{\partial^{2} \bar{g}_{i j}}{\partial x^{\alpha} \partial x^{\beta}}\right)_{x^{\gamma=0}}, \cdots
$$

There are similar expansions for $b^{i i}$,

$$
\bar{b}^{i j}=\delta_{j}^{i}+\epsilon \xi^{\alpha} b^{i j(\alpha)}+\frac{1}{2} \epsilon^{2} \xi^{\alpha} \xi^{\beta} b^{i j(\alpha \beta)}+\cdots,
$$

where the coefficients $b^{i j(\alpha)}, b^{i j(\alpha \beta)}, \ldots$ are defined by equations analogous to (7.6), and since the relations $\bar{g}_{j k} b^{i k}=\delta_{j}^{i}$ are true for all values of $\epsilon$ and $\xi^{\alpha}$, these coefficients are related to the coefficients in (7.5) by

$$
b^{i j(\alpha)}=-\bar{g}_{i j}^{(\alpha)}, \quad b^{i j(\alpha \beta)}=2 \bar{g}_{i k}^{(\alpha)} \bar{g}_{j k}^{(\beta)}-\bar{g}_{i j}^{(\alpha \beta)}, \cdots \cdot
$$

We note that $\bar{g}_{i j}$ is finite when $i=j$, and is of order $\epsilon$ when $i \neq j$.

It will be assumed that the components of the unknown metric tensor $\dot{g}_{i j}$ of the strained rod in equilibrium admit expansions in powers of $\epsilon$,

$$
g_{i j}=g_{i j(0)}+\epsilon g_{i j(1)}+\epsilon^{2} g_{i j(2)}+\cdots,
$$

the coefficients being regular functions of $x^{0}, \xi^{\alpha}$. From (4.1) it then follows that there are expansions for the strain tensor

$$
e_{i j}=e_{i j(0)}+\epsilon e_{i j(1)}+\cdots,
$$

and because of the stress-strain relations, for the reduced stress tensor,

$$
T_{i j}=T_{i j(0)}+\epsilon T_{i j(1)}+\cdots .
$$

To confine our attention to small strain, we must limit ourselves to those cases where 


$$
e_{i j(0)}=0 \text {. }
$$

Then, since strain and stress must vanish simultaneously to the same order of magnitude,

$$
T_{i j(0)}=0 .
$$

When we substitute in (4.1) the power series (7.5), (7.9) and (7.10) and equate coefficients of powers of $\epsilon$, we obtain, by use of (7.12),

$$
g_{i j(0)}=\delta_{j}^{i}, 2 e_{i j(1)}=g_{i j(1)}-\bar{g}_{i j}^{(\alpha)} \xi^{\alpha}, 2 e_{i j(2)}=g_{i j(2)}-\frac{1}{2} \bar{g}_{i j}^{(\alpha \beta)} \xi^{\alpha} \xi^{\beta}, \ldots
$$

These equations relate the coefficients in (7.9) and (7.10). By means of the stress-strain relations (4.3), we can also relate the coefficients in (7.10) and (7.11), or (7.9) and (7.11). Only the latter relations will be required. They appear in the following section (equations (8.10), (8.11)).

In (7.9), (7.10) and (7.11), the coefficients of $\epsilon^{0}$ are known. If for any problem we determine all the remaining coefficients, we shall have solved that problem exactly. If we determine only the coefficients up to and including those of $\epsilon^{n}$, we shall have obtained an approximate solution of the problem to within an error of order $\epsilon^{n+1}$, and the smaller $\epsilon$ (i.e., the thinner the rod), the better the approximation. In order to derive equations to be solved for the unknown coefficients in (7.9), (7.10) and (7.11), we shall express as power series in $\epsilon$ with coefficients independent of $\epsilon$ the left sides of the equations of equilibrium (5.1), the equations of compatibility (5.3) and the macroscopic equations of equilibrium (6.18) and (6.19), and then equate to zero the coefficients of the various powers of $\epsilon$. To this end, we must express as power series in $\epsilon$ the quantities $g^{i j}, F_{j k}^{i}, n_{i}, P_{i}, Q_{i}, \bar{\omega}^{i}, \omega^{i}$ and $e$. This will occupy the remainder of this section.

In view of (7.9), $g^{i j}$ can be expressed in the form

$$
g^{i j}=\delta_{j}^{i}+\epsilon g_{(1)}^{i j}+\epsilon^{2} g_{(2)}^{i j}+\cdots
$$

and since the relations

$$
g_{j k} g^{i k}=\delta_{j}^{i}
$$

are true for all values of $\epsilon$, it is found that

$$
\stackrel{i j}{g_{(1)}}=-g_{i j(1)}, \quad g_{(2)}^{i j}=g_{i k(1)} g_{j k(1)}-g_{i j(2)}, \cdots .
$$

Differentiation with respect to $x^{0}$ and $\xi^{\alpha}$ will be denoted by a subscript preceded by a comma. Thus

$$
\phi, 0=\frac{\partial \phi}{\partial x^{0}}, \quad \phi_{, \alpha}=\frac{\partial \phi}{\partial \xi^{\alpha}} .
$$


The Christoffel symbols of the second kind $F_{j k}^{i}$ are functions of $g^{i j}$ and the derivatives of $g_{i j}$ with respect to $x^{i}$. By substitution, they can thus be expressed in the form

$$
F_{j k}^{i}=F_{j k(0)}^{i}+\epsilon F_{j k(1)}^{i}+\cdots
$$

where

$$
\begin{aligned}
& 2 F_{00(0)}^{0}=0, \quad 2 F_{00(0)}^{\alpha}=-g_{00(1), \alpha}, \quad 2 F_{0 \alpha(0)}^{0}=g_{00(1), \alpha}, \\
& 2 F_{\alpha 0(0)}^{\beta}=g_{\beta 0(1), \alpha}-g_{0 \alpha(1), \beta}, \quad 2 F_{\alpha \beta(0)}^{0}=g_{\beta 0(1), \alpha}+g_{0 \alpha(1), \beta}, \\
& 2 F_{\alpha \beta(0)}^{\gamma}=g_{\beta \gamma(1), \alpha}+g_{\gamma \alpha(1), \beta}-g_{\alpha \beta(1), \gamma}, \\
& 2 F_{00(1)}^{0}=g_{00(1), 0}+g_{0 \alpha(1)} g_{00(1), \alpha}, \\
& 2 F_{0 \alpha(1)}^{0}=g_{00(2), \alpha}-g_{00(1)} g_{00(1), \alpha}-g_{0 \beta(1)}\left(g_{\beta 0(1), \alpha}-g_{0 \alpha(1), \beta}\right) .
\end{aligned}
$$

There are similar expressions for the remaining components of $F_{j k(1)}^{t}$, and for $F_{j k(2)}^{i}, F_{j k(3)}^{i}, \cdots$, which will not be required in the present investigation.

We shall now express as a power series in $\epsilon$ the unit covariant vector $n_{i}$ pointing along the outward normal to the lateral surface of the strained rod. The equation of the lateral surface of the rod is (7.4). Thus

$$
n_{0}=0, \quad n_{\alpha}=k f_{, \alpha},
$$

where $k^{-2}=g^{\alpha \beta} f_{, \alpha} f_{, \beta}$. In (7.15), $g^{\alpha \beta}$ is expressed as a power series in $\epsilon$. Thus $k$ and hence also $n_{i}$ can be expressed as a power series in $\epsilon$. We obtain

$$
n_{0}=0, \quad n_{\alpha}=n_{\alpha(0)}+\epsilon n_{\alpha(1)}+\cdots,
$$

where

$$
n_{\alpha(0)}=f_{, \alpha}\left(f_{, \beta} f_{, \beta}\right)^{-1 / 2}, \quad n_{\alpha(1)}=\frac{1}{2} f_{, \alpha}\left(f_{, \beta} f_{, \beta}\right)^{-3 / 2} g_{\gamma \delta(1)} f_{, \gamma} f_{, \delta}, \cdots .
$$

We note that if $d \xi^{\alpha}$ are the components of an arbitrary displacement on the periphery of a cross-section $N$, then $f_{, \alpha} d \xi^{\alpha}=0$, whence $f_{, 1} / d \xi^{2}=-f_{, 2} / d \xi^{1}$. Thus (7.23) can be written in the form

$$
n_{1(0)}=\frac{d \xi^{2}}{d \tau}, \quad n_{2(0)}=-\frac{d \xi^{1}}{d \tau}, \ldots,
$$

where

$$
(d \tau)^{2}=d \xi^{\alpha} d \xi^{\alpha} .
$$

The reaction across a cross-section of the strained rod is specified by a certain reduced force $P_{i}$ and a reduced couple $Q_{i}$ satisfying (6.1) and (6.2), respectively. An explicit evaluation of $P_{i}$ and $Q_{i}$ as power series in $\epsilon$ will now be made. The vectors $\lambda^{i}, \nu^{i}$ and $s \mu_{i}$ in (6.1) and (6.2) are given by (6.5), (6.6) and (6.10). They can thus be expressed as power series in $\epsilon$ by substituting for $x^{\alpha}, g_{i j}, g^{i j}, F_{j k}^{i}$ from (7.3), (7.9), (7.15) and (7.18). We then obtain 


$$
\begin{aligned}
\lambda^{i} & =\left(\lambda^{i}\right)_{C}-\epsilon \xi^{\alpha}\left(F_{\alpha k}^{i}\right)_{C}\left(\lambda^{k}\right)_{C}+O\left(\epsilon^{2}\right), \\
\nu^{0} & =1-\frac{1}{2} \epsilon g_{00(1)}+O\left(\epsilon^{2}\right), \quad \nu^{\alpha}=-\epsilon g_{\alpha 0(1)}+O\left(\epsilon^{2}\right), \\
s \mu_{0} & =\epsilon^{2}\left[\left(g_{0 \alpha(1)}\right)_{C} \xi^{\alpha}+\frac{1}{2}\left(g_{0 \alpha(1), \beta}\right)_{c} \xi^{\alpha \beta}\right]+O\left(\epsilon^{3}\right), \\
s \mu_{\alpha} & =\epsilon \xi^{\alpha}+\epsilon^{2}\left[\left(g_{\alpha \beta(1)}\right)_{C} \xi^{\beta}+\frac{1}{4}\left(2 g_{\alpha \beta(1), \gamma}+g_{\beta \gamma(1), \alpha}\right)_{c} \xi \xi^{\gamma}\right]+O\left(\epsilon^{3}\right),
\end{aligned}
$$

where $O\left(\epsilon^{r}\right)$ means terms of order $\epsilon^{r}$ and higher. The element of area $d S$ on $N$ is given by

$$
d S=\left(g^{\prime}\right)^{1 / 2} d x^{1} d x^{2}=\epsilon^{2}\left(g^{\prime}\right)^{1 / 2} d \xi^{1} d \xi^{2},
$$

where

$$
g^{\prime}=\left|g_{\alpha \beta}\right|=1+\epsilon g_{\alpha \alpha(1)}+O\left(\epsilon^{2}\right) .
$$

Of the quantities occurring on the right-hand sides of (6.1) and (6.2), $T_{i j}, \lambda^{i}, \nu^{i}, s \mu_{i}$ and $d S$ are expressed in (7.11), (7.25), (7.26), (7.27) and (7.28) as power series in $\epsilon$; by (7.9), $g=\left|g_{i j}\right|$ can be similarly expressed; and thus the entire right-hand sides of (6.1) and (6.2) can be expressed as power series in $\epsilon$. In the resulting equations, $\left(\lambda^{i}\right)_{c}$ appears in every term, and since $\left(\lambda^{i}\right)_{c}$ is an arbitrary unit vector at $C$, we conclude that

$$
\begin{aligned}
& P_{i}=\epsilon^{3} P_{i(3)}+\epsilon^{4} P_{i(4)}+\cdots, \\
& Q_{i}=\epsilon^{4} Q_{i(4)}+\epsilon^{5} Q_{i(5)}+\cdots,
\end{aligned}
$$

where

$$
\begin{aligned}
& P_{i(3)}=\int_{N} T_{0 i(1)} d \xi^{1} d \xi^{2}, \\
& P_{i(4)}=\int_{N}\left[T_{0 i(2)}+\frac{1}{2}\left(g_{\alpha \alpha(1)}-g_{00(1)}\right) T_{0 i(1)}\right. \\
& \left.-g_{\alpha 0(1)} T_{\alpha i(1)}-T_{0 j(1)} \xi^{\alpha}\left(F_{\alpha i(0)}^{j}\right)_{C}\right] d \xi^{1} d \xi^{2}, \\
& \text { (7.34) } \quad Q_{i(4)}=\int_{N} c^{i \alpha k \xi^{\alpha}} T_{k 0(1)} d \xi^{1} d \xi^{2}, \\
& Q_{i(5)}=\int_{N}\left[c^{j \alpha k} g_{j i(1)} \xi^{\alpha} T_{k 0(1)}-c^{j \alpha k}\left(F_{\beta i(0)}^{j}\right)_{c} \xi^{\beta} \xi^{\alpha} T_{k 0(1)}\right. \\
& +c^{i \alpha k}\left\{\left(g_{\alpha \beta(1)}\right)_{c} \xi^{\beta}+\frac{1}{4}\left(2 g_{\alpha \beta(1), \gamma}+g_{\beta \gamma(1), \alpha}\right) c^{\xi \beta} \xi^{\gamma}\right\} T_{k 0(1)} \\
& +c^{i \alpha k \xi^{\alpha}}\left\{T_{k 0(2)}-T_{k j(1)} g_{j 0(1)}\right\} \\
& \left.+c^{i 0 k}\left\{\left(g_{0 \alpha(1)}\right)_{C} \xi^{\alpha}+\frac{1}{2}\left(g_{0 \alpha+1), \beta}\right)_{c} \xi^{\alpha \xi^{\beta}}\right\} T_{k 0(1)}\right] d \xi^{1} d \xi^{2} .
\end{aligned}
$$

By (7.5), $\bar{g}=\left|\bar{g}_{i j}\right|$ can be expressed as a power series in $\epsilon$. Thus, by (2.8) we are able to express the rotation vector $\bar{\omega}^{i}$ of the unstrained rod in the form 


$$
\bar{\omega}^{i}=\frac{1}{2} c^{i \alpha k} \bar{g}_{0 k}^{(\alpha)} .
$$

By (7.9), $g=\left|g_{i j}\right|$ can also be expressed as a power series in $\epsilon$. Thus, by (3.7) we can express the rotation vector $\omega^{i}$ of the strained rod in the form

$$
\omega^{i}=\stackrel{i}{\omega_{(0)}}+\underset{\epsilon}{i}(1)+\cdots,
$$

where

$$
\begin{aligned}
& \stackrel{i}{\omega_{(0)}}=\frac{1}{2} c^{i \alpha k}\left(g_{0 k(1), \alpha}\right) c \\
& i_{(1)}^{i}=\frac{1}{2} c^{i \beta k}\left(g_{0 k(2), \beta}-\frac{1}{2} g_{j j(1)} g_{0 k(1), \beta}\right)_{C}+\frac{1}{2} c^{i 0 \beta}\left(g_{0 \beta(1), 0}\right)_{C} .
\end{aligned}
$$

By (3.9) we can also express $e$, the elongation per unit length of the line of centroids, in the form

$$
e=\epsilon e_{(1)}+\epsilon^{2} e_{(2)}+\cdots,
$$

where

$$
\begin{aligned}
& e_{(1)}=\frac{1}{2}\left(g_{00(1)}\right)_{c}, \\
& e_{(2)}=\frac{1}{2}\left(g_{00(2)}\right)_{C}-\frac{1}{8}\left(g_{00(1)}\right)_{C} .
\end{aligned}
$$

8. The fundamental equations. In this section, there will be developed equations for the determination of the unknown coefficients in the expansions (7.9), (7.10), (7.11) for $g_{i j}, e_{i j}, T_{i j}$, respectively.

The equations of equilibrium (5.1) can be written in the form

$$
g^{j k}\left(\frac{\partial T_{i k}}{\partial x^{j}}-F_{j k}^{l} T_{i l}-F_{i j}^{l} T_{k l}\right)=0 .
$$

All quantities in this equation are expressed in (7.11), (7.15) and (7.18) as power series in $\epsilon$. Hence we can express the left-hand side of $(8.1)$ as a power series in $\epsilon$, and since (8.1) is true for all small values of $\epsilon$, the coefficients of the various powers of $\epsilon$ must vanish identically. The coefficients of $\epsilon^{0}$ vanish if

$$
T_{i \alpha(1), \alpha}=0 .
$$

These are the first order equations of equilibrium. It is found that the coeffcients of $\epsilon$ vanish if

$$
\begin{aligned}
T_{i \alpha(2), \alpha} & +T_{i 0(1), 0}-g_{j \alpha(1)} T_{i j(1), \alpha}-g_{0 \alpha(1), \alpha} T_{i 0(1)} \\
& -\frac{1}{2}\left(2 g_{\gamma \alpha(1), \alpha}-g_{j j(1), \gamma}\right) T_{i \gamma(1)}-\frac{1}{2} \delta_{i}^{\alpha} g_{j k(1), \alpha} T_{j k(1)}=0 .
\end{aligned}
$$

These are the second order equations of equilibrium. Similarly there are equations of equilibrium of the third order, fourth order, and so on.

All quantities in the equations of compatibility (5.3) have also been expressed as power series in $\epsilon$. The left sides of the equations themselves can then be expressed as power series in $\epsilon$, and the coefficients of the various 
powers of $\epsilon$ must vanish identically. The coefficients of $\epsilon^{-1}$ give rise to the first order equations of compatibility

$$
\begin{aligned}
g_{00(1), \alpha \beta} & =0, \\
g_{01(1), 22}-g_{02(1), 12} & =0, \\
g_{02(1), 11}-g_{01(1), 21} & =0, \\
2 g_{12(1), 12}-g_{11(1), 22}-g_{22(1), 11} & =0,
\end{aligned}
$$

while the coefficients of $\epsilon^{0}$ give rise to the second order equations of compatibility

$$
g_{00(2), \alpha \beta}-g_{0 \alpha(1), 0 \beta}-g_{0 \beta(1), 0 \alpha}-\frac{1}{2} g_{00(1), \alpha} g_{00(1), \beta}
$$

$$
-\frac{1}{2}\left(g_{0 \gamma(1), \alpha}-g_{0 \alpha(1), \gamma}\right)\left(g_{0 \gamma(1), \beta}-g_{0 \beta(1), \gamma}\right)
$$$$
-\frac{1}{2} g_{00(1), \gamma}\left(g_{\beta \gamma(1), \alpha}+g_{\gamma \alpha(1), \beta}-g_{\alpha \beta(1), \gamma}\right)=0 \text {, }
$$

$g_{01(2), 22}-g_{02(2), 12}+g_{22(1), 01}-g_{12(1), 02}+g_{00(1), 1} g_{02(1), 2}$

$$
\begin{aligned}
& +\frac{1}{2} g_{\alpha \alpha(1), 2}\left(g_{02(1), 1}-g_{01(1), 2}\right)-\frac{1}{2} g_{00(1), 2}\left(g_{02(1), 1}+g_{01(1), 2}\right)=0, \\
g_{02(2), 11} & -g_{01(2), 21}+g_{11(1), 02}-g_{21(1), 01}+g_{00(1), 2} g_{01(1), 1} \\
& +\frac{1}{2} g_{\alpha \alpha(1), 1}\left(g_{01(1), 2}-g_{02(1), 1}\right)-\frac{1}{2} g_{00(1), 1}\left(g_{01(1), 2}+g_{02(1), 1}\right)=0, \\
2 g_{12(2), 12} & -g_{11(2), 22}-g_{22(2), 11}-2 g_{01(1), 1} g_{02(1), 2} \\
& +\frac{1}{2}\left(g_{2 \gamma(1), 1}+g_{\gamma 1(1), 2}-g_{12(1), \gamma}\right)\left(g_{2 \gamma(1), 1}+g_{\gamma 1(1), 2}-g_{12(1), \gamma}\right) \\
& +\frac{1}{2}\left(g_{02(1), 1}+g_{01(1), 2}\right)^{2}-\frac{1}{2}\left(2 g_{1 \gamma(1)}-g_{11(1), \gamma}\right)\left(2 g_{2 \gamma(1), 2}-g_{22(1), \gamma}\right)=0 .
\end{aligned}
$$

Similarly we can write down equations of compatibility of the third order, the fourth order, and so on.

By replacing $e_{i j}$ in the stress-strain relations (4.3) by $\frac{1}{2}\left(g_{i j}-\bar{g}_{i j}\right)$ and substituting for $\bar{g}_{i j}, g_{i j}$ and $T_{i j}$ from (7.5), (7.9) and (7.11) with $T_{i j(0)}=0$ as per (7.13), in the same way we deduce stress-strain relations of the first order,

$$
g_{i j(1)}=\bar{g}_{i j}^{(\alpha)} \xi^{\alpha}-2 \sigma \delta_{j}^{i} T_{k k(1)}+2(1+\sigma) T_{i j(1)},
$$

and stress-strain relations of the second order,

$$
\begin{aligned}
g_{i j(2)}= & \frac{1}{2} \bar{g}_{i j}^{(\alpha \beta)} \xi^{\alpha} \xi^{\beta}-2 \sigma \delta_{j}^{i} T_{k k(2)}+2(1+\sigma) T_{i j(2)} \\
& +\delta_{j}^{i}\left[2 \sigma g_{k l(1)} T_{k l(1)}-\left(2 \sigma_{1}+\sigma_{2}-\sigma_{3}\right) T_{k k(1)} T_{l l(1)}\right. \\
& \left.+\left(\sigma_{2}-\sigma_{3}\right) T_{k l(1)} T_{k l(1)}\right] \\
& -2 \sigma T_{k k(1)} g_{i j(1)}+2\left(1+\sigma+\sigma_{2}-\sigma_{3}\right) T_{k k(1)} T_{i j(1)} \\
& +2\left[2(1+\sigma)^{2}+\sigma_{3}\right] T_{i k(1)} T_{j k(1)} .
\end{aligned}
$$

In the stress-strain relations (4.3), quantities of order $\left(e_{i j}\right)^{3}$ were neglected. If such quantities had been retained, it would be possible to write down stressstrain relations of the third order. Similarly stress-strain relations of higher orders could be found. 
The boundary conditions on the lateral surface of the rod are (5.4). By substitution in (5.4) from (7.11), (7.13), (7.15) and (7.22), we can convert the left sides into power series in $\epsilon$. It is found that the coefficients of $\epsilon$ vanish if

$$
T_{i \beta(1)} n_{\beta(0)}=0 .
$$

These are the first order boundary conditions. Similarly the coefficients of $\boldsymbol{\epsilon}^{2}$ vanish if

$$
T_{i \beta(2)} n_{\beta(0)}-T_{i j(1)} g_{j \beta(1)} n_{\beta(0)}+T_{i \beta(1)} n_{\beta(1)}=0 .
$$

These are the second order boundary conditions. In the same way there could be written down boundary conditions of the third order, of the fourth order, and so on.

All quantities in the macroscopic equations of equilibrium (6.18) and (6.19) have been expressed in $\$ 7$ as power series in $\epsilon$. By substitution, the left sides of these equations can then be expressed as power series in $\epsilon$ and the coefficients of the various powers of $\epsilon$ equated to zero. The lowest power of $\epsilon$ occurring is $\epsilon^{3}$, and its coefficients vanish if

$$
\begin{aligned}
\frac{d P_{i(3)}}{d x^{0}}-\left(F_{i 0(0)}^{j}\right)_{C} P_{j(3)} & =0, \\
P_{\alpha(3)} & =0 .
\end{aligned}
$$

These are the first order macroscopic equations of equilibrium. The coefficients of $\epsilon^{4}$ vanish if

$$
\begin{array}{r}
\frac{d P_{i(4)}}{d x^{0}}-\left(F_{i 0(0)}^{j}\right)_{C} P_{j(4)}-\left(F_{0 i(1)}^{0}\right)_{C} P_{0(3)}=0, \\
\frac{d Q_{i(4)}}{d x^{0}}-\left(F_{i 0(0)}^{j}\right) c Q_{j(4)}+c^{i \alpha 0}\left(g_{\alpha 0(1)}\right)_{C} P_{0(3)}+c^{i 0 \alpha} P_{\alpha(4)}=0,
\end{array}
$$

and these are the second order macroscopic equations of equilibrium. The coefficients of $\epsilon^{5}$ vanish if

$$
\begin{aligned}
& \frac{d P_{i(5)}}{d x^{0}}-\left(F_{i 0(0)}^{j}\right)_{C} P_{j(5)}-\left(F_{i 0(1)}^{j}\right)_{C} P_{j(4)}-\left(F_{0 i(2)}^{0}\right)_{C} P_{0(3)}=0, \\
& \frac{d Q_{i(5)}}{d x^{0}}-\left(F_{i 0(0)}^{j}\right) c Q_{j(5)}-\left(F_{i 0(1)}^{j}\right) c Q_{j(4)}+c^{i 0 \alpha} P_{\alpha(5)} \\
& +\left(g_{i \alpha(1)}-\frac{1}{2} \delta_{i}^{\alpha} g_{n n(1)}\right)_{C} c^{\alpha 0 \beta} P_{\beta(4)}+c^{i k l}\left(g_{k 0(1)}\right)_{C} P_{l(4)} \\
& +\left(\delta_{i}^{\alpha} g_{\beta 0(2)}+g_{i \alpha(1)} g_{\beta 0(1)}-\frac{1}{2} \delta_{i}^{\alpha} g_{\beta 0(1)} g_{n n(1)}\right)_{c} c^{\alpha \beta 0} P_{0(3)}=0 \text {, }
\end{aligned}
$$

where the required components of $F_{j k(r)}^{i}$ are given in (7.19) and (7.20). These are the third order macroscopic equations of equilibrium. Similarly there can be written down macroscopic equations of equilibrium of higher orders. 
The fundamental equations listed in the previous part of this section are to be solved in the following manner:

I. $T_{i j}$ is the reduced stress tensor and $g_{i j}$ the metric tensor of the strained rod. In (7.11) and (7.9), they are expressed as power series in $\epsilon$, the first terms $T_{i j(0)}$ and $g_{i j(0)}$ being given by (7.13) and (7.14). If any problem in thin rods is to be considered to the first approximation, it is only necessary to determine $T_{i j(1)}$ and $g_{i j(1)}$, and this is done by solving the first order stress-strain relations (8.10) with the first order equations of equilibrium and compatibility (8.2), (8.4), (8.5) and (8.6), subject to the first order boundary conditions (8.12) over the sides of the rod. These solutions for $T_{i j(1)}$ and $g_{i j(1)}$ will contain arbitrary functions of $x^{0}$. By substitution for $T_{i j(1)}$ and $g_{i j(1)}$ in (7.32) and (7.34) it is then possible to express $P_{i(3)}$ and $Q_{i(4)}$ in terms of these unknown functions of $x^{0}$, which can then be determined by means of the first and second order macroscopic equations of equilibrium (8.14), (8.15), (8.16) and (8.17).

II. If any problem is to be considered to the second approximation, it is necessary to determine $T_{i j(2)}$ and $g_{i j(2)}$ in addition to $T_{i j(1)}$ and $g_{i j(1)}$ already found as above. This is done by means of the second order stress-strain relations (8.11) and the second order equations of equilibrium and compatibility (8.3), (8.7), (8.8) and (8.9), subject to the second order boundary conditions (8.13). These solutions for $T_{i j(2)}$ and $g_{i j(2)}$ will also contain arbitrary functions of $x^{0}$. By substitution for $T_{i j(2)}$ and $g_{i j(2)}$ in (7.33) and (7.35) it is possible to express $P_{i(4)}$ and $Q_{i(5)}$ in terms of these unknown functions of $x^{0}$, which can then be determined by means of the third order macroscopic equations of equilibrium, (8.18) and (8.19).

III. By continuing in the same way, we can consider any problem to a third approximation by determining $T_{i j(3)}$ and $g_{i j(3)}$, to a fourth approximation by determining $T_{i j(4)}$ and $g_{i j(4)}$, and so on.

9. The first approximation: the stress. As indicated in $\S 8, \mathrm{I}$, just above, the first order equations of equilibrium

$$
T_{i \alpha(1), \alpha}=0,
$$

and the first order equations of compatibility

$$
\begin{aligned}
g_{00(1), \alpha \beta} & =0, \\
\gamma_{01(1), 22}-g_{02(1), 12} & =0, \\
g_{02(1), 11}-g_{01(1), 21} & =0, \\
2 g_{12(1), 12}-g_{11(1), 22}-g_{22(1), 11} & =0,
\end{aligned}
$$

with the first order boundary conditions

$$
T_{i \beta(1)} n_{\beta(0)}=0,
$$

are to be solved for $T_{i j(1)}$ and $g_{i j(1)}$, which are themselves related by the first order stress-strain relations 


$$
g_{i j(1)}=\bar{g}_{i j}^{(\alpha)} \xi^{\alpha}-2 \sigma \delta_{j}^{i} T_{k k(1)}+2(1+\sigma) T_{i j(1)} .
$$

To solve equations of this nature, recourse is usually made to SaintVenant's hypothesis, which states that these equations imply that $T_{\alpha \beta(1)}=0$. It is not necessary to make this hypothesis here, as in Appendix A it is proved that, of necessity,

$$
T_{\alpha \beta(1)}=0 .
$$

This constitutes a proof of Saint-Venant's hypothesis in the case of thin rods displaced finitely.

From (9.2) it follows that

$$
g_{00(1)}=\left(g_{00}^{(\gamma)}+A_{\gamma}\right) \xi^{\gamma}+B,
$$

where $A_{\gamma}$ and $B$ are arbitrary functions of $x^{0}$. When $i=j=0,(9.6)$ then gives

$$
T_{00(1)}=\frac{1}{2}\left(A_{\alpha} \xi^{\alpha}+B\right) \text {. }
$$

We now solve (9.1) and (9.3) for $T_{0 \alpha(1)}$ and $g_{0 \alpha(1)}$. From (9.3),

$$
g_{01(1), 2}-g_{02(1), 1}=D^{\prime},
$$

where $D^{\prime}$ is an arbitrary function of $x^{0}$. By (9.6), this gives

$$
T_{01(1), 2}-T_{02(1), 1}=-D /(1+\sigma),
$$

where $D=-\frac{1}{2}\left(D^{\prime}-\bar{g}_{01}^{(2)}+\bar{g}_{02}^{(1)}\right)$. From (9.11) and (9.1) with $i=0$ it follows that

$$
T_{01(1)}=\frac{D}{2(1+\sigma)} \chi_{, 2}, \quad T_{02(1)}=-\frac{D}{2(1+\sigma)} \chi_{, 1},
$$

where $\chi$ is a function of $\xi^{\alpha}$ only, satisfying

$$
\chi, \alpha \alpha=-2 .
$$

By (7.24), the boundary condition (9.5) with $i=0$ is satisfied if

$$
\chi=0
$$

on the lateral surface of the rod.

From (2.2), $\bar{g}_{\alpha \beta}^{(\gamma)}=0$, and thus the collected results are

$$
\begin{aligned}
& T_{\alpha \beta(1)}=0, \quad T_{0 \alpha(1)}=\frac{D}{2(1+\sigma)} c_{\alpha \beta} \chi, \beta, \quad T_{00(1)}=\frac{1}{2}\left(A_{\alpha} \xi^{\alpha}+B\right), \\
& g_{\alpha \beta(1)}=-\sigma \delta_{\beta}^{\alpha}\left(A_{\gamma} \xi^{\gamma}+B\right), \\
& g_{0 \alpha(1)}=\bar{g}_{0 \alpha}^{(\gamma)} \xi^{\gamma}+D c_{\alpha \beta} \chi, \beta, \quad g_{00(1)}=\bar{g}_{00}^{(\gamma)} \xi^{\gamma}+A_{\gamma} \xi^{\gamma}+B,
\end{aligned}
$$

$c_{\alpha \beta}$ being a permutation symbol and $A_{\alpha}, B$ and $D$ arbitrary functions of $x^{0}$. By means of (9.16), the leading coefficients in the expressions (7.37), and (7.40) for $\omega^{i}$ and $e$ can now be expressed in the form 


$$
\begin{aligned}
& \underset{\omega_{(0)}^{0}}{0}=\frac{1}{2} c_{\alpha \beta} \bar{g}_{0 \beta}^{(\alpha)}+D, \\
& \underset{(0)}{\alpha}=\frac{1}{2} c_{\alpha \beta}\left(\bar{g}_{00}^{(\beta)}+A_{\beta}\right), \\
& \omega_{(1)}^{0}=\frac{1}{2} c_{\beta \gamma}\left(g_{0 \gamma(2), \beta}\right)_{C}-\frac{1}{4}(1-2 \sigma) B\left(2 D+c_{\beta \gamma} \bar{g}_{0 \gamma}^{(\beta)}\right), \\
& \underset{(1)}{\alpha}=\frac{1}{2} c_{\alpha \beta}\left(g_{00(2), \beta}\right)_{C}-\frac{1}{4}(1-2 \sigma) B c_{\alpha \beta}\left(\bar{g}_{00}^{(\beta)}+A_{\beta}\right)+\frac{1}{2} \frac{d D}{d x^{0}}(\chi, \alpha)_{C},
\end{aligned}
$$

$$
e_{(1)}=\frac{1}{2} B \text {. }
$$

10. The first approximation: the macroscopic equations of equilibrium. The arbitrary functions of $x^{0}$ occurring in (9.15) and (9.16) are now to be determined in the manner outlined in $\S 8, \mathrm{I}$. We find that

$$
\begin{gathered}
P_{0(3)}=\frac{1}{2} B A, \quad P_{\alpha(3)}=0, \\
Q_{0(4)}=D T, \quad Q_{1(4)}=\frac{1}{2} A_{2} I_{1}, \quad Q_{2(4)}=-\frac{1}{2} A_{1} I_{2},
\end{gathered}
$$

where

$$
\begin{aligned}
A=\int_{N} d \xi^{1} d \xi^{2}, & I_{1}=\int_{N}\left(\xi^{2}\right)^{2} d \xi^{1} d \xi^{2}, \\
T=\frac{1}{1+\sigma} \int_{N} \chi d \xi^{1} d \xi^{2}, & I_{2}=\int_{N}\left(\xi^{1}\right)^{2} d \xi^{1} d \xi^{2},
\end{aligned}
$$

$N$ being a cross-section of the strained rod. We note that

$$
A \epsilon^{2}=A^{\prime}, \quad I_{\alpha} \epsilon^{4}=I_{\alpha}^{\prime}, \quad T E \epsilon^{4}=T^{\prime},
$$

where $A^{\prime}$ is the area of $\bar{N}$ (Figure 1), $I_{\alpha}^{\prime}$ are the principal moments of inertia of $\bar{N}, T^{\prime}$ is the torsional rigidity of a straight rod with $\bar{N}$ as cross-section, $E$ is Young's modulus and $\epsilon$ is defined in (7.1).

The first order macroscopic equations of equilibrium (8.14) and (8.15). are satisfied if

$$
\begin{aligned}
\frac{d B}{d x^{0}} & =0, \\
\left(\bar{g}_{00}^{(\alpha)}+A_{\alpha}\right) B & =0 .
\end{aligned}
$$

Thus we must consider in turn the three classes of problems which arise according as the arbitrary functions $A_{\alpha}$ and $B$ of $x^{0}$ satisfy the following conditions:

(i) $B=0,\left(\bar{g}_{00}^{(\alpha)}+A_{\alpha}\right)\left(\bar{g}_{00}^{(\alpha)}+A_{\alpha}\right) \neq 0$,

(ii) $B=$ constant $\neq 0, \bar{g}_{00}^{(\alpha)}+A_{\alpha}=0$,

(iii) $B=0, \bar{g}_{00}^{(\alpha)}+A_{\alpha}=0$.

By (9.17) and (9.19) we see that, in Class (i) the curvature of $M$ (the strained line of centroids) is finite $\left(O\left(\epsilon^{0}\right)\right)$ and the elongation per unit length of $M$ is 
relatively small $\left(O\left(\epsilon^{2}\right)\right)$. In Class (ii), the curvature is small $(O(\epsilon))$ and the elongation per unit length is relatively large $(O(\epsilon))$. In Class (iii), the curvature is small $(O(\epsilon))$ and the elongation per unit length is relatively small $\left(O\left(\epsilon^{2}\right)\right)$.

Classes (ii) and (iii) deal with the straightening of thin rods by terminal force-systems (see §13). By "straightening" we mean "reducing the curvature of the strained line of centroids from order $\epsilon^{0}$ to order $\epsilon . "$

Class (i). $B=0,\left(\bar{g}_{00}^{(\alpha)}+A_{\alpha}\right)\left(\bar{g}_{00}^{(\alpha)}+A_{\alpha}\right) \neq 0$. (elongation per unit length relatively small, curvature finite).

The equations (10.1) and (10.2) reduce to

$$
\begin{array}{ll}
P_{i(3)}=0, & Q_{0(4)}=D T, \\
Q_{1(4)}=\frac{1}{2} A_{2} I_{1}, & Q_{2(4)}=-\frac{1}{2} A_{1} I_{2},
\end{array}
$$

and the macroscopic equations of equilibrium of the second order (8.16) and (8.17) take the form

$$
\begin{aligned}
\frac{d P_{0(4)}}{d x^{0}}+\frac{1}{2}\left(\bar{g}_{00}^{(\alpha)}+A_{\alpha}\right) P_{\alpha(4)} & =0 \\
\frac{d P_{\alpha(4)}}{d x^{0}}-\frac{1}{2}\left(\bar{g}_{00}^{(\alpha)}+A_{\alpha}\right) P_{0(4)}-\frac{1}{2}\left(2 D+\bar{g}_{02}^{(1)}-\bar{g}_{01}^{(2)}\right) c_{\alpha \beta} P_{\beta(4)} & =0 \\
\frac{d Q_{0(4)}}{d x^{0}}+\frac{1}{2}\left(\bar{g}_{00}^{(\alpha)}+A_{\alpha}\right) Q_{\alpha(4)} & =0 \\
\frac{d Q_{\alpha(4)}}{d x^{0}}-\frac{1}{2}\left(\bar{g}_{00}^{(\alpha)}+A_{\alpha}\right) Q_{0(4)}-\frac{1}{2}\left(2 D+\bar{g}_{02}^{(1)}-\bar{g}_{01}^{(2)}\right) c_{\alpha \beta} Q_{\beta(4)}-c_{\alpha \beta} P_{\beta(4)} & =0
\end{aligned}
$$

Thus-

IV. In Class (i) the external terminal force-systems, which act only on the ends of the rod, are of order $\epsilon^{4}$ or higher. The nine unknown functions $A_{\alpha}, D, P_{i(4)}, Q_{i(4)}$, of $x^{0}$, can be determined from (10.7) and (10.8). Once $A_{\alpha}$ and $D$ have been found in this way, the principal parts of the rotation $\omega_{(0)}^{1}$, the reduced stress tensor $T_{i j(1)}$, the metric tensor of the strained rod $g_{i j(1)}$ and the strain tensor $e_{i j(1)}$ can be found from (9.17), (9.15), (9.16) and (7.14).

From (7.36) and (9.17), we note that

$$
\begin{aligned}
D & =\stackrel{0}{\omega_{(0)}}-\overline{\bar{\omega}}^{0}, \\
A_{\alpha} & =-2 c_{\alpha \beta}\left(\omega_{(0)}^{\beta}-\bar{\omega}^{\beta}\right),
\end{aligned}
$$

and consequently (10.7) and (10.8), when expressed entirely in terms of the small quantities, become

$$
\begin{aligned}
& Q_{0}=\left(\omega^{0}-\bar{\omega}^{0}\right) T^{\prime} / E, \\
& Q_{1}=\left(\omega^{1}-\bar{\omega}^{1}\right) I_{1}^{\prime}, \quad Q_{2}=\left(\omega^{2}-\bar{\omega}^{2}\right) I_{2}^{\prime},
\end{aligned}
$$




$$
\frac{d P_{i}}{d x^{0}}+c^{i j k} \omega^{i} P_{k}=0, \quad \frac{d Q_{i}}{d x^{0}}+c^{i j k} \omega^{j} Q_{k}-c^{0 i j} P_{j}=0,
$$

where $E P_{i}$ and $E Q_{i}$ are respectively the force and couple statically equivalent to the reaction across a cross-section of the strained rod, $E, T^{\prime}$ and $I_{\alpha}{ }^{\prime}$ are as defined in (10.4), $\bar{\omega}^{i}$ and $\omega^{i}$ are respectively the rotations in the unstrained and strained rods, $c^{i j k}$ is a permutation symbol and $x^{0}$ is the arc length measured along the unstrained line of centroids. We note that (10.10) and (10.11) are a system of nine equations from which we are to determine the nine unknowns $P_{i}, Q_{i}$ and $\omega^{i}$.

In previous treatments of the finite displacement of thin rods the equations (10.10) and (10.11) have been deduced, but those treatments do not go past this point and are thus equivalent to the first stage of the systematic $\epsilon$-method of approximation developed for problems of Class (i) only.

Class (ii). $B=$ constant $\neq 0, \bar{g}_{00}^{(\alpha)}+A_{\alpha}=0$ (elongation per unit length relatively large, curvature small).

The equations (10.1) and (10.2) reduce to

$$
\begin{gathered}
P_{0(3)}=\frac{1}{2} B A, \quad P_{\alpha(3)}=0, \\
Q_{0(4)}=D T, \quad Q_{1(4)}=-\frac{1}{2} \bar{g}_{00}^{(2)} I_{1}, \quad Q_{2(4)}=\frac{1}{2} \bar{g}_{00}^{(1)} I_{2} .
\end{gathered}
$$

The second order macroscopic equations of equilibrium (8.16) and (8.17) are found to involve $\left(g_{00(2), \alpha}\right)_{C}$ which, by the second order equations of compatibility (8.7), have the forms

$$
\left(g_{00(2), \alpha}\right)_{C}=2 M_{\alpha},
$$

$M_{\alpha}$ being an arbitrary function of $x^{0}$. By integration of these second order macroscopic equations of equilibrium, and by (10.12), it is then found that

(10.14)

$$
\begin{aligned}
& P_{0(3)}=\text { constant }, \quad P_{0(4)}=\text { constant, } \\
& P_{1(4)}=-\frac{1}{2} \bar{g}_{00,0}^{(1)} I_{2}+\frac{1}{4}\left(2 D+. \bar{g}_{02}^{(1)}-\bar{g}_{01}^{(2)}\right) \bar{g}_{00}^{(2)} I_{1}+(\chi, 2)_{C} P_{0(3)} D,
\end{aligned}
$$

(10.15)

$$
\begin{aligned}
P_{2(4)}= & -\frac{1}{2} \bar{g}_{00,0}^{(2)} I_{1}+\frac{1}{4}\left(-2 D+\bar{g}_{01}^{(2)}-\bar{g}_{02}^{(1)}\right) \bar{g}_{00}^{(1)} I_{2}-(\chi, 1)_{C} P_{0(3)} D, \\
Q_{0(4)}= & D T=\text { constant, } \\
Q_{1(4)}= & -\frac{1}{2} \bar{g}_{00}^{(2)} I_{1}, \quad Q_{2(4)}=\frac{1}{2} \bar{g}_{00}^{(1)} I_{2}, \\
M_{1} P_{0(3)}= & -\frac{1}{2} \bar{g}_{00,00}^{(1)} I_{2}+\frac{1}{2}\left(2 D+\bar{g}_{02}^{(1)}-\bar{g}_{01}^{(2)}\right) \bar{g}_{00,0}^{(2)} I_{1} \\
& +\frac{1}{8}\left(2 D+\bar{g}_{02}^{(1)}-\bar{g}_{01}^{(2)}\right) \bar{g}_{00}^{(1)} I_{2}+\frac{1}{4}\left(\bar{g}_{02,0}^{(1)}-\bar{g}_{01,0}^{(2)}\right) \bar{g}_{00}^{(2)} I_{1}, \\
M_{2} P_{0(3)}= & -\frac{1}{2} \bar{g}_{00,00}^{(2)} I_{1}+\frac{1}{2}\left(-2 D+\bar{g}_{01}^{(2)}-\bar{g}_{02}^{(1)}\right) \bar{g}_{00,0}^{(1)} I_{2} \\
& +\frac{1}{8}\left(-2 D+\bar{g}_{01}^{(2)}-\bar{g}_{02}^{(1)}\right) \bar{g}_{00}^{(2)} I_{1}+\frac{1}{4}\left(\bar{g}_{01,0}^{(2)}-\bar{g}_{02,0}^{(1)}\right) \bar{g}_{00}^{(1)} I_{2} .
\end{aligned}
$$


The arbitrary functions $M_{\alpha}$ of $x^{0}$ in (10.13) are given by (10.15). We note that $D$ must be constant. From (9.17) and (9.18) we obtain

$$
\stackrel{0}{\omega_{(0)}}=\frac{1}{2} c_{\alpha \beta} \bar{g}_{0 \beta}^{(\alpha)}+D, \quad \stackrel{\alpha}{\omega_{(1)}}=c_{\alpha \beta} M_{\alpha},
$$

giving the principal parts of the twist and curvature. Thus-

V. In Class (ii), there act on the ends of the rod arbitrary equal and opposite forces of order $\epsilon^{3}$, arbitrary twisting couples of order $\epsilon^{4}$ and particular bending couples of order $\epsilon^{4}$ given by (10.14) and depending only on the configuration of the unstrained rod. The principal parts of the reaction across a cross-section satisfy (10.14). The principal parts of the twist and curvature of the strained line of centroids, of order $\epsilon^{0}$ and $\epsilon$ respectively, are given by (10.16). The principal parts of the reduced stress tensor $T_{i j}$, the metric tensor $g_{i j}$ and the strain tensor $e_{i j}$ can be found from (9.15), (9.16) and (7.14), respectively.

Class (iii). $B=0, \bar{g}_{00}^{(\alpha)}+A_{\alpha}=0$ (elongation per unit length relatively small, curvature small).

The equations of equilibrium (10.1) and (10.2) reduce to

$$
\begin{gathered}
P_{i(3)}=0 \\
Q_{0(4)}=D T, \quad Q_{1(4)}=-\frac{1}{2} \bar{g}_{00}^{(2)} I_{1}, \quad Q_{2(4)}=\frac{1}{2} \bar{g}_{00}^{(1)} I_{2} .
\end{gathered}
$$

The three equations in (10.18) and the six second order macroscopic equations of equilibrium (8.16) and (8.17) are to be solved for the seven unknowns $Q_{i(4)}, P_{i(4)}, D$. Solutions exist only if

$$
\begin{aligned}
& D=0, \\
& -\bar{g}_{00,00}^{(1)} I_{2}+\frac{1}{2}\left(\bar{g}_{02,0}^{(1)}-\bar{g}_{01,0}^{(2)}\right) \bar{g}_{00}^{(2)} I_{1} \\
& +\left(\bar{g}_{02}^{(1)}-\bar{g}_{01}^{(2)}\right) \bar{g}_{00,0}^{(2)} I_{1}+\frac{1}{4}\left(\bar{g}_{02}^{(1)}-\bar{g}_{01}^{(2)}\right)^{2} \bar{g}_{00}^{(1)} I_{2}=0, \\
& -\bar{g}_{00,00}^{(2)} I_{1}+\frac{1}{2}\left(\bar{g}_{01,0}^{(2)}-\bar{g}_{02,0}^{(1)}\right) \bar{g}_{00}^{(1)} I_{2} \\
& +\left(\bar{g}_{01}^{(2)}-\bar{g}_{02}^{(1)}\right) \bar{g}_{00,0}^{(1)} I_{2}+\frac{1}{4}\left(\bar{g}_{01}^{(2)}-\bar{g}_{02}^{(1)}\right)^{2} \bar{g}_{00}^{(2)} I_{1}=0 ;
\end{aligned}
$$

(10.20) can also be written in the form

$$
\begin{aligned}
& \left(\bar{\omega}^{0}{ }_{0} \bar{\omega}^{1}+2 \bar{\omega}^{0} \bar{\omega}^{1}, 0\right) I_{1} / I_{2}+\bar{\omega}_{, 00}^{2}-\left(\bar{\omega}^{0}\right)^{2} \bar{\omega}^{2}=0, \\
& \left(\bar{\omega}_{, 0}^{0} \bar{\omega}^{2}+2 \bar{\omega}^{0} \bar{\omega}^{2}, 0\right) I_{2} / I_{1}-\bar{\omega}_{, 00}^{1}+\left(\bar{\omega}^{0}\right)^{2} \bar{\omega}^{1}=0,
\end{aligned}
$$

in which $I_{1} / I_{2}$ is the ratio of the principal moments of inertia of the crosssection, $\bar{\omega}^{i}$ is the rotation vector defined in $\S 2$, and the subscript 0 preceded by a comma denotes differentiation with respect to $x^{0}$, the arc length of the line of centroids. When (10.21) are satisfied, the solutions of (10.17), (10.18), (10.19), (8.16) and (8.17) are 
$(10.22)$

$$
\begin{aligned}
& P_{0(4)}=\text { constant } \\
& P_{1(4)}=-\frac{1}{2} \bar{g}_{00,0}^{(1)} I_{2}+\frac{1}{4}\left(\bar{g}_{02}^{(1)}-\bar{g}_{01}^{(2)}\right) \bar{g}_{00}^{(2)} I_{1}, \\
& P_{2(4)}=-\frac{1}{2} \bar{g}_{00,0}^{(2)} I_{1}+\frac{1}{4}\left(\bar{g}_{01}^{(2)}-\bar{g}_{02}^{(1)}\right) \bar{g}_{00}^{(1)} I_{2}, \\
& Q_{0(4)}=0 \\
& Q_{1(4)}=-\frac{1}{2} \bar{g}_{00}^{(2)} I_{1}, \\
& Q_{2(4)}=\frac{1}{2} \bar{g}_{00}^{(1)} I_{2},
\end{aligned}
$$

and we then have

$$
\stackrel{0}{\omega_{(1)}}=\bar{\omega}^{0}, \quad \stackrel{\alpha}{\omega_{(1)}}=c_{\alpha \beta} M_{\alpha},
$$

for the principal parts of the twist and curvature. Thus-

VI. Class (iii) deals only with those rods which satisfy (10.20). There act on the ends of the rod arbitrary forces of order $\epsilon^{4}$, arbitrary twisting couples of order $\epsilon^{5}$ or higher, and particular bending couples of order $\epsilon^{4}$ given by (10.22) and depending only on the configuration of the unstrained rod. The principal parts of the reaction across a cross-section are given by (10.22). From (10.23) we see that the principal part of the twist $\omega^{0}$ (of order $\epsilon^{0}$ ) is equal to the twist $\bar{\omega}^{0}$ of the unstrained rod, and the principal parts of the curvature $\omega^{\alpha}$ (of order $\epsilon$ ) depend on $M_{\alpha}$ which are arbitrary functions of $x^{0}$ and cannot be determined from the equations of the first approximation. The principal parts of the reduced stress tensor $T_{i j}$, the metric tensor $g_{i j}$ and the strain tensor $e_{i j}$ are given by (9.15), (9.16) and (7.14) with $B=D=0$,

$$
A_{\alpha}=-\bar{g}_{00}^{(\alpha)} \text {. }
$$

If $\bar{\omega}^{0}=0$ (i.e., there is no twist in the unstrained rod), (10.20) reduce to

$$
\bar{g}_{00,00}^{(\alpha)}=0 .
$$

By integrating, we obtain

$$
\bar{g}_{00}^{(\alpha)}=V_{\alpha} x^{0}+W_{\alpha},
$$

$V_{\alpha}, W_{\alpha}$ being arbitrary constants. Thus by (7.36),

$$
\bar{\omega}^{\alpha}=\frac{1}{2} c_{\alpha \beta}\left(V_{\beta} x^{0}+W_{\beta}\right),
$$

i.e., the curvature of the unstrained line of centroids is directly proportional to its arc length.

11. The second approximation: the stress. As indicated in $\$ 8, \mathrm{II}$, the second order equations of equilibrium (8.3) and the second order equations of compatibility (8.7), (8.8), (8.9) with the second order boundary conditions (8.13) are to be solved for $T_{i j(2)}$ and $g_{i j(2)}$, which are themselves related by the second order stress-strain relations (8.11). These equations are very complicated. We shall now determine $T_{i j(2)}$ and $g_{i j(2)}$ when the cross-section of the unstrained rod is a circle of radius $d$, the unstrained line of centroids is a 
plane curve with curvature proportional to its arc length, and the force-systems acting on the ends of the rod are such that the line of centroids remains in its original plane. The problem is thus two-dimensional and belongs to Class (iii). We choose the axis of $x^{1}$ perpendicular to the plane of the line of centroids. Thus from (10.3) we find that

$$
A=\pi R^{2}, \quad I_{1}=I_{2}=\frac{1}{4} \pi R^{4},
$$

where $\epsilon R=d$. Also

$$
\begin{aligned}
\bar{\omega}^{0} & =0, \quad \bar{\omega}^{1}=\frac{1}{2}\left(V_{2} x^{0}+W_{2}\right), \quad \bar{\omega}^{2}=0, \\
\bar{g}_{\alpha \beta} & =\delta_{\beta}^{\alpha}, \quad \bar{g}_{0 \alpha}=0, \quad \bar{g}_{00}=\left[1+\frac{1}{2} x^{2}\left(V_{2} x^{0}+W_{2}\right)\right]^{2}, \\
P_{1} & =Q_{0}=Q_{2}=0, \\
P_{0(4)} & =p, \quad P_{2(4)}=-\frac{1}{8} \pi R^{4} V_{2}, \quad Q_{1(4)}=-\frac{1}{8} \pi R^{4}\left(V_{2} x^{0}+W_{2}\right), \\
T_{\alpha \beta(1)} & =0, \quad T_{0 \alpha(1)}=0, \quad T_{00(1)}=-\frac{1}{2} \xi^{2}\left(V_{2} x^{0}+W_{2}\right), \\
g_{\alpha \beta(1)} & =\sigma \delta_{\beta}^{\alpha} \xi^{2}\left(V_{2} x^{0}+W_{2}\right), \quad g_{0 \alpha(1)}=0, \quad g_{00(1)}=0,
\end{aligned}
$$

$p$ being an arbitrary constant and $V_{2}$ and $W_{2}$ known constants. We find that the second order equations of equilibrium (8.3) reduce to

$$
T_{0 \alpha(2), \alpha}-\frac{1}{2} V_{2} \xi^{2}=0, \quad T_{\alpha \beta(2), \beta}=0,
$$

the second order equations of compatibility (8.7), (8.8) and (8.9) to

$$
\begin{gathered}
g_{00(2), \alpha \beta}=0, \\
g_{01(2), 22}-g_{02(2), 12}=0, \quad g_{02(2), 11}-g_{01(2), 21}+\sigma V_{2}=0, \\
2 g_{12(2), 12}-g_{11(2), 22}-g_{22(2), 11}+\sigma^{2}\left(V_{2} x^{0}+W_{2}\right)^{2}=0,
\end{gathered}
$$

the second order boundary conditions $(8.13)$ to

$$
T_{i \beta(2)} n_{\beta(0)}=0,
$$

and the second order stress-strain relations (8.11) to

$$
\begin{aligned}
g_{\alpha \beta(2)}= & -2 \sigma \delta_{\beta}^{\alpha} T_{i i(2)}+2(1+\sigma) T_{\alpha \beta(2)} \\
& +\left(\sigma^{2}-\frac{1}{2} \sigma_{1}\right) \delta_{\beta}^{\alpha}\left(\xi^{2}\right)^{2}\left(V_{2} x^{0}+W_{2}\right)^{2}, \\
g_{0 \alpha(2)}= & 2(1+\sigma) T_{0 \alpha(2)}, \\
g_{00(2)}= & -2 \sigma T_{i i(2)}+2(1+\sigma) T_{00(2)}+\frac{1}{4} \Lambda\left(\xi^{2}\right)^{2}\left(V_{2} x^{0}+W_{2}\right)^{2},
\end{aligned}
$$

where

$$
\Lambda=7+10 \sigma+4 \sigma^{2}-2 \sigma_{1}+2 \sigma_{2},
$$

and $\sigma_{1}, \sigma_{2}$ and $\sigma$ are the elastic constants introduced in $\$ 4$. By solving these equations we find that 


$$
T_{00(2)}=\frac{1}{32}\left[\frac{\Gamma \sigma}{1-\sigma}\left(R^{2}-2 \xi^{\alpha} \xi^{\alpha}\right)-4 \Lambda \xi^{2} \xi^{2}\right]\left(V_{2} x^{0}+W_{2}\right)^{2}+M_{2} \xi^{2}+\frac{1}{2} L,
$$

(11.13)

$$
\begin{aligned}
T_{\alpha \beta(2)}= & \frac{\Gamma}{64(1-\sigma)}\left[\left(R^{2}-\xi^{\gamma} \xi^{\gamma}\right) \delta_{\beta}^{\alpha}-2 c_{\alpha \gamma} c_{\beta \delta} \xi^{\gamma} \xi^{\delta}\right]\left(V_{2} x^{0}+W_{2}\right)^{2}, \\
T_{0 \alpha(2)}= & \frac{1+2 \sigma}{8(1+\sigma)} \xi^{1} c_{\alpha \beta} \xi^{\beta} V_{2}-\frac{3+2 \sigma}{16(1+\sigma)}\left(R^{2}-\xi^{\gamma} \xi^{\gamma}\right) \delta_{\alpha}^{2} V_{2}, \\
g_{00(2)}= & 2 M_{2} \xi^{2}+L, \\
g_{\alpha \beta(2)}= & \left\{\frac{(1+\sigma)}{32(1-\sigma)}\left[(1-2 \sigma) R^{2}-(1-4 \sigma) \xi \gamma \xi^{\gamma}\right]\right. \\
& \left.+\frac{1}{4}\left(\Lambda \sigma+4 \sigma^{2}-2 \sigma_{1}\right) \xi^{2} \xi^{2}\right\} \delta_{\beta}^{\alpha}\left(V_{2} x^{0}+W_{2}\right)^{2}-\sigma \delta_{\beta}^{\alpha}\left(2 M_{2} \xi^{2}+L\right) \\
& -\frac{\Gamma(1+\sigma)}{16(1-\sigma)} c_{\alpha \gamma} c_{\beta \delta} \xi^{\gamma} \xi^{\delta}\left(V_{2} x^{0}+W_{2}\right)^{2}, \\
g_{0 \alpha(2)}= & \frac{1}{4}(1+2 \sigma) \xi^{1} c_{\alpha \beta} \xi^{\beta} V_{2}-\frac{1}{8}(3+2 \sigma)\left(R^{2}-\xi^{\gamma} \xi^{\gamma}\right) \delta_{\alpha}^{2} V_{2} ;
\end{aligned}
$$

$c_{\alpha \beta}$ is a permutation symbol; $M_{2}$ and $L$ are arbitrary functions of $x^{0} ; V_{2}$ and $W_{2}$ are the constants occurring in (11.2); $R=d / \epsilon, d$ being the radius of the cross-section of the unstrained rod; $\sigma, \sigma_{1}, \sigma_{2}$ and $\sigma_{3}$ are elastic constants, $\Lambda$ is given by (11.12) and

$$
\Gamma=\left(\Lambda \sigma+2 \sigma^{2}-2 \sigma_{1}\right) /(1+\sigma) .
$$

By (10.23), the principal part of the curvature of the strained line of centroids is given by

$$
\omega_{(1)}^{1}=M_{2} .
$$

Also, by (7.42) the principal part of the elongation per unit length of the line of centroids is given by

$$
e_{(2)}=\frac{1}{2} L .
$$

12. The second approximation: the macroscopic equations of equilibrium. The next step is the determination of the arbitrary functions $M_{2}$ and $L$ of $x^{0}$ occurring in (11.13) and (11.14) by means of the third order macroscopic equations of equilibrium (8.18) and (8.19). Now (7.33) and (7.35) reduce to

$$
\begin{aligned}
& P_{0(4)}=\frac{1}{2} \pi R^{2} L-\frac{1}{32} \pi R^{4}(\Lambda+4 \sigma)\left(V_{2} x^{0}+W_{2}\right)^{2}, \\
& P_{2(4)}=-\frac{1}{8} \pi R^{4} V_{2}, \\
& Q_{1(5)}=\frac{1}{4} \pi R^{4} M_{2},
\end{aligned}
$$

and by comparing (12.1) with (11.5), we see that 


$$
L=\frac{1}{16} \pi R^{2}(\Lambda+4 \sigma)\left(V_{2} x^{0}+W_{2}\right)^{2}+2 p \pi^{-1} R^{-2},
$$

$p$ being an arbitrary constant. The third order macroscopic equations of equilibrium (8.18) and (8.19) reduce to

$$
P_{0(5), 0}+M_{2} P_{2(4)}=0, \quad P_{2(5), 0}-M_{2} P_{0(4)}=0, \quad Q_{1(5), 0}-P_{2(5)}=0,
$$

and by solving (12.1) and (12.3), we find that

$$
\begin{aligned}
P_{0(4)} & =p, \quad P_{2(4)}=-\frac{1}{8} \pi R^{4} V_{2}, \quad Q_{1(4)}=-\frac{1}{8} \pi R^{4}\left(V_{2} x^{0}+W_{2}\right), \\
P_{0(5)} & =V_{2} u \cosh n x^{0}+V_{2} v n^{-1} \sinh n x^{0}+t, \\
P_{2(5)} & =2 n^{2} u \cosh n x^{0}+2 n v \sinh n x^{0}, \\
Q_{1(5)} & =2 n u \sinh n x^{0}+2 v \cosh n x^{0}, \\
M_{.2} & =8 \pi^{-1} R^{-4}\left(u n \sinh n x^{0}+v \cosh n x^{0}\right),
\end{aligned}
$$

where $p, t, u$ and $v$ are arbitrary constants and

$$
n=2 R^{-2}(p / \pi)^{1 / 2} .
$$

We assign $P_{0(4)}, P_{0(5)}, P_{2(5)}$ and $Q_{1(5)}$ arbitrarily at one end of the rod and hence determine $p, t, u$ and $v$.

From (11.16) and (11.17) we find that the principal parts of the curvature and the elongation per unit length are given by

$$
\begin{aligned}
& {\stackrel{1}{\omega_{(1)}}}^{1}=8 \pi^{-1} R^{-4}\left(u n \sinh n x^{0}+v \cosh n x^{0}\right), \\
& e_{(2)}=\frac{1}{32} R^{4}(\Lambda+4 \sigma)\left(V_{2} x^{0}+W^{2}\right)^{2}+p \pi^{-1} R^{-2} .
\end{aligned}
$$

13. The straightening of naturally curved thin rods by terminal forcesystems. A thin rod is said to be "straightened" when the curvature of the line of centroids is reduced from order $\epsilon^{0}$ (finite) to order $\epsilon$ (small). In $\$ 10$ it is seen how all problems in which thin rods undergo finite displacement with small strain can be divided into three classes. Classes (ii) and (iii) deal exclusively with "straightening," and will be considered in turn.

Straightening as a problem of Class (ii). The theory pertaining to problems of Class (ii) is developed in $\$ 10$, from which we obtain the result:

VII. Any thin rod with uniform cross-section and finite curvature can be straightened without undergoing finite strain by terminal force-systems consisting of arbitrary equal and opposite forces of order $\epsilon^{3}$ and particular bending couples of order $\epsilon^{4}$. The first approximation yields the residue curvature of the strained line of centroids. If the curvature of the unstrained line of centroids vanishes at the ends, no terminal bending couples are necessary. Further, if the twist and rate of change of curvature both vanish at the ends of the rod, the lines of action of the two forces acting one on each end will coincide and be tangent to the strained line of centroids at its ends.

As an example of a problem of Class (ii), we shall now straighten the rod 
shown in Figure 4. The unstrained line of centroids $\bar{M}$ is that part of an ellipse lying between the ends of the major axis, $a$ and $b$ are the semi-axes of this ellipse and rectangular cartesian coordinates $x$ and $y$ are taken as shown. The cross-section is assumed to be symmetrical with respect to the plane of $\bar{M}$,

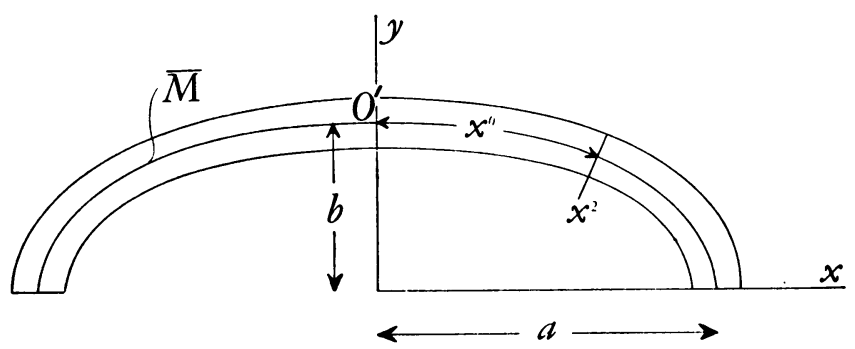

FIG. 4. An unstrained rod; the line of centroids $\bar{M}$ is the arc of an ellipse lying between the ends of the major axis

the axis of $x^{1}$ is taken perpendicular to this plane and $x^{0}$ is the arc length of $\bar{M}$ measured from its middle point $O^{\prime}$. If the equation of $\bar{M}$ is written in the form

$$
x=a \operatorname{sn} \zeta, \quad y=b \operatorname{cn} \zeta,
$$

then

$$
x^{0}=a \int_{0}^{\zeta} \operatorname{dn}^{2} \zeta d \zeta
$$

$\zeta$ being a parameter which vanishes at $O^{\prime}$, and $\operatorname{sn} \zeta$, cn $\zeta$ and $\operatorname{dn} \zeta$ the Jacobian elliptic functions of modulus $k\left(=\left(1-b^{2} / a^{2}\right)^{1 / 2}\right)$. We then have

$$
\bar{\omega}^{0}=0, \quad \bar{\omega}^{1}=a^{-2} b / \mathrm{dn}^{3} \zeta, \quad \bar{\omega}^{2}=0,
$$

$\bar{\omega}^{i}$ being the rotation vector introduced in $\S 2\left(\bar{\omega}^{1}\right.$ is now the curvature of $\left.\bar{M}\right)$. From (7.36) we then find that

$$
\bar{g}_{02}^{(1)}=\bar{g}_{01}^{(2)}, \quad \bar{g}_{00}^{(1)}=0, \quad \bar{g}_{00}^{(2)}=-a^{-2} b / \mathrm{dn}^{3} \zeta,
$$

whence (10.14), (10.15) and (10.16) reduce to

$$
\begin{aligned}
P_{0(3)} & =\text { constant }, \quad P_{2(4)}=3 I_{1} a^{-3} b k^{2} \operatorname{sn} \zeta \mathrm{cn} \zeta / \mathrm{dn}^{6} \zeta, \\
Q_{1(4)} & =I_{1} a^{-2} b / \mathrm{dn}^{3} \zeta, \\
\omega_{(1)}^{1} & =\frac{3 I_{1} k^{2}}{P_{0(3)} a^{8}}\left(\frac{\mathrm{cn}^{2} \zeta-\mathrm{sn}^{2} \zeta}{\operatorname{dn}^{7} \zeta}+6 k^{2} \frac{\operatorname{sn}^{2} \zeta \mathrm{cn}^{2} \zeta}{\operatorname{dn}^{9} \zeta}\right) .
\end{aligned}
$$

Thus the principal part of the reaction across a general cross-section of the strained rod consists of a tension $P_{0}^{\prime}\left(=E P_{0(3)} \epsilon^{3}\right)$, a shearing force $P_{2}^{\prime}$ $\left(=E P_{2(4)} \epsilon^{4}\right)$ and a bending couple $Q_{1}^{\prime}\left(=E Q_{1_{(4)}} \epsilon^{4}\right)$, whence 


$$
\begin{aligned}
& P_{0}^{\prime}=\text { an arbitrary constant of order } \epsilon^{3}, \\
& P_{2}^{\prime}=3 E I_{1}^{\prime} a^{-3} b k^{2} \operatorname{sn} \zeta \mathrm{cn} \zeta / \mathrm{dn}^{6} \zeta, \\
& Q_{1}^{\prime}=E I_{1}^{\prime} a^{-2} b / \mathrm{dn}^{3} \zeta,
\end{aligned}
$$

$I_{1}^{\prime}$ being the moment of inertia of the unstrained cross-section about an axis through its centroid and perpendicular to the plane of $\bar{M}$, and $\zeta$ a parameter related by (13.2) to the arc length $x^{0}$ of the unstrained rod. Also, the principal part of the curvature of the strained line of centroids is $\epsilon \omega_{(1)}^{1}$ or

$$
\frac{3 E I_{1}^{\prime} b k^{2}}{P_{0}^{\prime} a^{8}}\left(\frac{\mathrm{cn}^{2} \zeta-\mathrm{sn}^{2} \zeta}{\mathrm{dn}^{7} \zeta}+6 k^{2} \frac{\mathrm{sn}^{2} \zeta \mathrm{cn}^{2} \zeta}{\mathrm{dn}^{9} \zeta}\right) .
$$

At the right-hand end of the rod, sn $\zeta=1$, cn $\zeta=0, \operatorname{dn} \zeta=b / a$, and thus $P_{2}^{\prime}=0, Q_{1}^{\prime}=E I_{1}^{\prime} a b^{-2}$. Thus the force-system acting on the right-hand end of the rod consists of an arbitrary force $P_{0}^{\prime}$ of order $\epsilon^{3}$ and a particular bending couple, as shown in Figure 5. From symmetry, a similar force-system acts on the other end of the rod. Further, since $P_{2}^{\prime}=0$ at the ends of the rod, the lines of action of the two forces $P_{0}^{\prime}$ coincide and are tangent to the strained line of centroids at its ends.

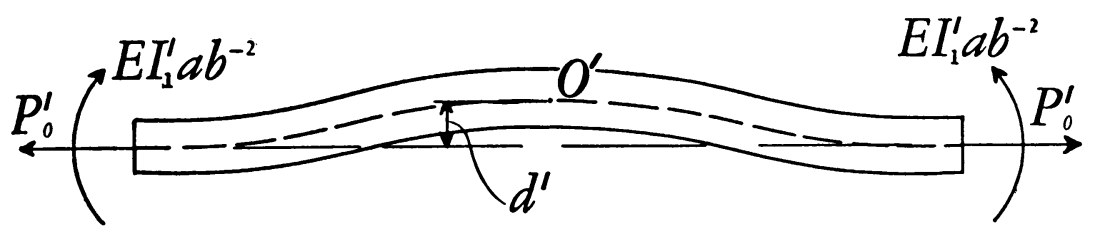

FIG. 5. The rod in Figure 4, straightened

At $O^{\prime}, \operatorname{dn} \zeta=1$ and hence $Q_{1}^{\prime}=E I_{1}^{\prime} a^{-2} b$. Thus by taking the moment about $O^{\prime}$ of the forces and couples acting on the right-hand half of the rod, we find that the distance $d^{\prime}$ (Figure 5) is

$$
d^{\prime}=\frac{E I_{1}^{\prime}\left(a^{3}-b^{3}\right)}{P^{\prime} a^{2} b^{2}} .
$$

Straightening as a problem of Class (iii). As mentioned previously, all problems of Class (iii) deal with the straightening of thin rods by terminal forcesystems. From the theory developed in $\$ \S 10-12$ for problems of this class, it can be conclude that-

VIII. Any thin rod of uniform cross-section and finite curvature, and satisfying (10.21), can be straightened without undergoing finite strain by means of terminal force-systems consisting of an arbitrary force and a particular bending couple, both of order $\epsilon^{4}$. The residue curvature of the strained line of centroids can be found only by recourse to the second approximation.

As an example of a problem of Class (iii), we shall now straighten the rod shown in Figure 6. The cross-section is a circle of radius $d$. The unstrained line 
of centroids $\bar{M}$ is a semicircle of radius $c$ and $x^{0}$ is its arc length measured from its middle point $O^{\prime}$. The $x^{1}$-axis is taken perpendicular to the plane of $\bar{M}$. Thus

$$
\bar{\omega}^{0}=0, \quad \bar{\omega}^{1}=-1 / c, \quad \bar{\omega}^{2}=0,
$$

$\bar{\omega}^{i}$ being the rotation vector introduced in $\$ 2$. Hence by comparison with (11.2),

$$
V_{1}=V_{2}=W_{1}=0, \quad W_{2}=-2 / c,
$$

and since the strained line of centroids $M$ is to be a plane curve symmetrical

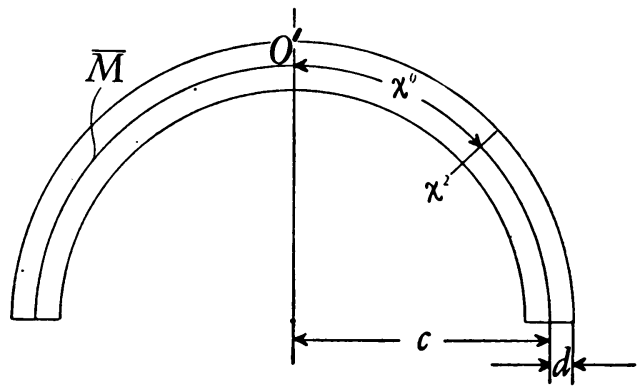

Fig. 6. An unstrained rod; the cross-section is circular and the line of centroids $\bar{M}$ is a semicircle

about its middle point $O^{\prime}$ (Figure 7), we find that (12.4), (12.7) and (12.8) reduce to

$$
\begin{aligned}
P_{0(4)} & =p, \quad Q_{1(4)}=\frac{1}{4} \pi R^{4} / c, \\
P_{0(5)} & =t, \quad P_{2(5)}=2 v n \sinh n x^{0}, \quad Q_{1(5)}=2 v \cosh n x^{0}, \\
\omega_{(1)}^{1} & =\frac{8 v}{\pi R^{4}} \cosh n x^{0}, \\
e_{(2)} & =(\Lambda+4 \sigma) \frac{R^{2}}{8 c^{2}}+\frac{p}{\pi R^{2}},
\end{aligned}
$$

where $p$ is an arbitrary constant and $n=2\left(p / \pi R^{4}\right)^{1 / 2}$. Thus the reaction across a cross-section of the strained rod consists of a normal force $P_{0}^{\prime}+P_{0}^{\prime \prime}$ $\left(P_{0}^{\prime}=E P_{0(4)} \epsilon^{4}=E p \epsilon^{4}, P_{0}^{\prime \prime}=E P_{0(5)} \epsilon^{5}\right)$, a shearing force $P_{2}^{\prime \prime}\left(=E P_{2(5)} \epsilon^{5}\right)$, and a bending couple $Q_{1}^{\prime}+Q_{1}^{\prime \prime}\left(Q_{1}^{\prime}=E Q_{1(4)} \epsilon^{4}, Q_{1}^{\prime \prime}=E Q_{1(5)} \epsilon^{5}\right)$; and

$$
\begin{aligned}
P_{0}^{\prime} & =\text { an arbitrary constant of order } \epsilon^{4}, \\
P_{0}^{\prime \prime} & =\text { an arbitrary constant of order } \epsilon^{5}, \\
P_{2}^{\prime \prime} & =2 E v^{\prime \prime} \sinh n x^{0}, \\
Q_{1}^{\prime} & =\frac{1}{4} \pi E d^{4} / c, \quad Q_{1}^{\prime \prime}=2 E v^{\prime \prime} \cosh n x^{0},
\end{aligned}
$$


where $n=2\left(P_{0}^{\prime} / \pi E d^{4}\right)^{1 / 2}$ and $v^{\prime \prime}$ is an arbitrary constant $\left(=\epsilon^{5} v\right)$. In (13.15), the quantities with a single prime are of order $\epsilon^{4}$, while those with a double prime are of order $\epsilon^{5}$. The strained line of centroids $M$ has a curvature $\omega^{1}$ $\left(=\epsilon \omega_{(1)}^{1}\right)$ given by

$$
\omega^{1}=\frac{8 v^{\prime \prime}}{\pi d^{4}} \cosh n x^{0},
$$

and an elongation per unit length $e\left(=\epsilon^{2} e_{(2)}\right)$ given by

$$
e=(\Lambda+4 \sigma) \frac{d^{2}}{8 c^{2}}+\frac{P_{0}^{\prime}}{\pi E d^{4}} .
$$

Figure 7 shows the straightened rod. From the consideration just above of the reaction across a cross-section, we see that there must act on the ends

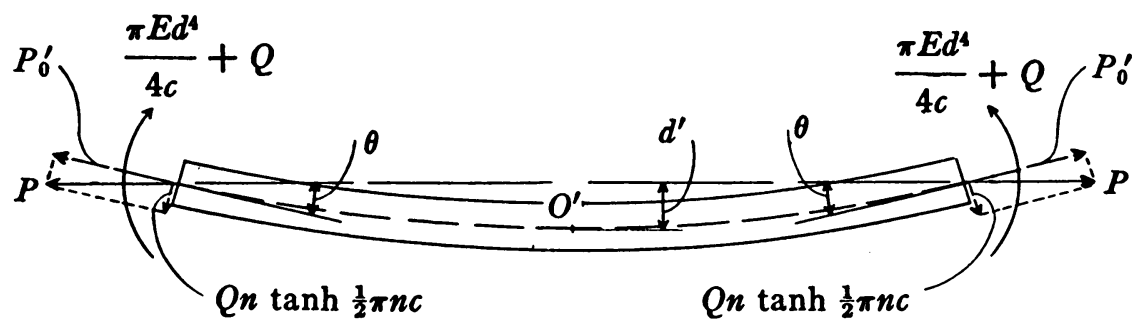

FIG. 7. The rod in Figure 6, straightened

of the rod equal and opposite forces $P$ of order $\epsilon^{4}$ and bending couples

$$
\frac{1}{4} \pi E d^{4} / c+Q
$$

as shown, $\frac{1}{4} \pi E d^{4} / c$ being of order $\epsilon^{4}$ and $Q$ of order $\epsilon^{5}$. Further, since $x^{0}= \pm \frac{1}{2} \pi c$ at the ends of the rod,

$$
\begin{gathered}
v^{\prime \prime}=Q /\left(2 E \cosh \frac{1}{2} \pi n c\right), \\
P \cos \theta=P_{0}^{\prime}, \quad P \sin \theta=Q n \tanh \frac{1}{2} \pi n c,
\end{gathered}
$$

$\theta$ being the small angle shown in Figure 7 . Since $\theta$ is small, we set

$$
\sin \theta=\theta, \quad \cos \theta=1 .
$$

Thus $P=P_{0}^{\prime}$ approximately, and

$$
\begin{aligned}
n & =2\left(P / \pi E d^{4}\right)^{1 / 2}, \\
\theta & =\frac{Q}{P} n \tanh \frac{1}{2} \pi n c, \\
e & =(\Lambda+4 \sigma) \frac{d^{2}}{8 c^{2}}+\frac{P}{\pi E d^{4}} .
\end{aligned}
$$


From (13.13) the curvature of the strained line of centroids is then

$$
\omega^{1}=\frac{4 Q}{\pi d^{4}} \frac{\cosh n x^{0}}{\cosh \frac{1}{2} \pi n c} .
$$

At $O^{\prime}, x^{0}=0$ and hence

$$
Q_{1}^{\prime \prime}=Q \operatorname{sech} \frac{1}{2} \pi n c .
$$

Thus by taking the moment about $O^{\prime}$ of the forces and couples acting on the right-hand half of the rod, we find that the distance $d^{\prime}$ (Figure 7) is

$$
d^{\prime}=\frac{Q}{P}\left(1-\operatorname{sech} \frac{1}{2} \pi n c\right) .
$$

If $P$ vanishes (i.e., no external forces are applied to the ends of the rod), then $n=0$ and

$$
\begin{gathered}
\theta=\lim _{P \rightarrow 0}\left(\frac{Q}{P} n \tanh \frac{1}{2} \pi n c\right)=2 Q c /\left(E d^{4}\right), \\
e=(\Lambda+4 \sigma) d^{2} /\left(8 c^{2}\right), \\
\omega^{1}=4 Q /\left(\pi E d^{4}\right) .
\end{gathered}
$$

In this case the line of centroids of the strained rod is an arc of a circle of radius

$$
\frac{1}{4} \pi E d^{4} / Q .
$$

14. Remarks on the experimental determination of elastic constants. The stress-strain relations (4.3) used in this paper contain five independent elastic constants $E, \sigma, \sigma_{1}, \sigma_{2}, \sigma_{3} ; E$ is Young's modulus and $\sigma$ is Poisson's ratio. Both $E$ and $\sigma$ can be determined for any isotropic elastic material by simple experiments involving the stretching and twisting of a straight rod. In this section, we shall see how a relation between $\sigma_{1}$ and $\sigma_{2}$ can be determined experimentally by straightening the thin rod shown in Figure 6 . The straightened rod is shown in Figure 7. When terminal bending couples alone of magnitude $\frac{1}{4} \pi E d^{4} / c$ act on the rod, the elongation per unit length of the line of centroids is given by (13.26). By actually measuring this elongation, we can thus obtain an experimental value for $\Lambda+4 \sigma$ for the particular material of which the rod is constructed. Now $\sigma$ is known for most elastic materials. Thus $\Lambda$ can also be found. By (11.12), this gives a relation between $\sigma_{1}$ and $\sigma_{2}$. In order to determine the arithmetical values of the three elastic constants $\sigma_{1}, \sigma_{2}$ and $\sigma_{3}$ occurring in the stress-strain relations (4.3), it would be necessary to devise additional experimental methods of obtaining two additional relations between $\sigma_{1}, \sigma_{2}$ and $\sigma_{3}$.

The author would like to take this opportunity to thank Dr. J. L. Synge both for suggesting the problem considered in this paper and for much assistance in the development of the mathematical theory. 
Appendix A. Proof of Saint-Venant's hypothesis for a thin rod displaced finitely $\left({ }^{12}\right)$. We are to show that, given

$$
\begin{gathered}
T_{\alpha \beta(1), \beta}=0, \\
g_{00(1), \alpha \beta}=0, \\
2 g_{12(1), 12}-g_{11(1), 22}-g_{22(1), 11}=0, \\
g_{\alpha \beta(1)}=\bar{g}_{\alpha \beta}^{(r)} \xi^{\gamma}-2 \sigma \delta_{\beta}^{\alpha} T_{k k(1)}+2(1+\sigma) T_{\alpha \beta(1)},
\end{gathered}
$$

with the boundary conditions

$$
T_{\alpha \beta(1)} n_{\beta(0)}=0,
$$

it is necessary that $T_{\alpha \beta(1)}=0$.

From (A.2),

$$
g_{00(1)}=A_{\alpha}^{\prime} \xi^{\alpha}+B,
$$

$A_{\alpha}^{\prime}$ and $B$ being arbitrary functions of $x^{0}$. Then by (A.4),
$T_{00(1)}=\sigma T_{\alpha \alpha(1)}+\frac{1}{2}\left(A_{\alpha} \xi^{\alpha}+B\right)$,$$
T_{i i(1)}=(1+\sigma) T_{\alpha \alpha(1)}+\frac{1}{2}\left(A_{\alpha} \xi^{\alpha}+B\right),
$$

$A_{\alpha}$ being an arbitrary function of $x^{0}$ such that $A_{\alpha}=-\bar{g}_{00}^{(\alpha)}+A_{\alpha}^{\prime}$. Substitution in (A.3) for $g_{\alpha \beta(1)}$ from (A.4) and then for $T_{i i(1)}$ from (A.8) gives

$$
T_{11(1), 22}+T_{22(1), 11}-2 T_{12(1), 12}=\sigma T_{\alpha \alpha(1), \beta \beta} .
$$

It is necessary to show that there exist functions $u_{\alpha}\left(x^{0}, \xi^{\beta}\right)$ and a constant $\sigma^{\prime}$ such that

$$
\frac{1}{2}\left(u_{\alpha, \beta}+u_{\beta, \alpha}\right)=\left(1+\sigma^{\prime}\right) T_{\alpha \beta(1)}-\sigma^{\prime} \delta_{\beta}^{\alpha} T_{\gamma \gamma(1)} .
$$

These are satisfied when $\alpha=\beta=1, \alpha=\beta=2$ if

$$
\begin{aligned}
& u_{1}=\int_{0}^{\xi^{1}}\left[\left(1+\sigma^{\prime}\right) T_{11(1)}-\sigma^{\prime} T_{\gamma \gamma(1)}\right] d \xi^{1}+u_{1}^{\prime}\left(x^{0}, \xi^{2}\right), \\
& u_{2}=\int_{0}^{\xi^{2}}\left[\left(1+\sigma^{\prime}\right) T_{22(1)}-\sigma^{\prime} T_{\gamma \gamma(1)}\right] d \xi^{2}+u_{2}^{\prime}\left(x^{0}, \xi^{1}\right),
\end{aligned}
$$

and the third equation in (A.10) is satisfied if

$$
\begin{aligned}
u_{1,2}^{\prime}+u_{2,1}^{\prime} & +\int_{0}^{\xi^{1}}\left[\left(1+\sigma^{\prime}\right) T_{11(1), 2}-\sigma^{\prime} T_{\gamma \gamma(1), 2}\right] d \xi^{1} \\
& +\int_{0}^{\xi^{2}}\left[\left(1+\sigma^{\prime}\right) T_{22(1), 1}-\sigma^{\prime} T_{\gamma \gamma(1), 1}\right] d \xi^{2}=2\left(1+\sigma^{\prime}\right) T_{12(1)} .
\end{aligned}
$$

(12) The method of proof when the displacement is small has been indicated by Goodier. See J. N. Goodier, loc. cit., $\$ 4$. 
Now

$$
\begin{aligned}
& \int_{0}^{\xi^{1}} \int_{0}^{\xi^{2}} {\left[\left(1+\sigma^{\prime}\right) T_{11(1), 22}-\sigma^{\prime} T_{\gamma \gamma(1), 22}\right] d \xi^{1} d \xi^{2} } \\
&= \int_{0}^{\xi^{1}}\left[\left(1+\sigma^{\prime}\right) T_{11(1), 2}-\sigma^{\prime} T_{\gamma \gamma(1), 2}\right] d \xi^{1} \\
&-\int_{0}^{\xi^{1}}\left[\left(1+\sigma^{\prime}\right) T_{11(1), 2}-\sigma^{\prime} T_{\gamma \gamma(1), 2}\right] \xi^{2}=0 \\
&
\end{aligned}
$$

From this and a similar expression derived by interchange of the indices 1 and 2 , it is seen that (A.12) takes the form

$$
\begin{aligned}
u_{1,2}^{\prime}+u_{2,1}^{\prime} & +\int_{0}^{\xi^{1}} \int_{0}^{\xi^{2}}\left[\left(1+\sigma^{\prime}\right)\left(T_{11(1), 22}+T_{22(1), 11}\right)-\sigma^{\prime} T_{\gamma \gamma(1), \beta \beta}\right] d \xi^{1} d \xi^{2} \\
& +\int_{0}^{\xi^{1}}\left[\left(1+\sigma^{\prime}\right) T_{11(1), 2}-\sigma^{\prime} T_{\gamma \gamma(1), 2}\right] \xi_{\xi^{2}=0} d \xi^{1} \\
& +\int_{0}^{\xi^{2}}\left[\left(1+\sigma^{\prime}\right) T_{22(1), 1}-\sigma^{\prime} T_{\gamma \gamma(1), 1}\right]_{\xi^{1}=0} d \xi^{2}=2\left(1+\sigma^{\prime}\right) T_{12(1)} .
\end{aligned}
$$

If

$$
\sigma^{\prime}=\sigma /(1-\sigma)
$$

where $\sigma$ is Poisson's ratio, then by (A.9) the double integral in (A.14) becomes

$$
\begin{aligned}
& 2 \int_{0}^{\xi^{1}} \int_{0}^{\xi^{2}}\left(1+\sigma^{\prime}\right) T_{12(1), 12} d \xi^{1} d \xi^{2} \\
& \quad=2\left(1+\sigma^{\prime}\right)\left(T_{12(1)}-\left[T_{12(1)}\right]_{\xi^{1}=0}-\left[T_{12(1)}\right]_{\xi^{2}=0}+\left[T_{12(1)}\right]_{\xi^{1}=\xi^{2}=0}\right) .
\end{aligned}
$$

Thus (A.14) takes the form

$$
u_{1,2}^{\prime}+u_{2,1}^{\prime}=G_{0}\left(x^{0}\right)+G_{1}\left(x^{0}, \xi^{1}\right)+G_{2}\left(x^{0}, \xi^{2}\right) .
$$

Since the variables $\xi^{\alpha}$ have been separated, $u_{\alpha}^{\prime}$ can be found. Thus $u_{\alpha}$ exist satisfying (A.10), the constant $\sigma^{\prime}$ having the value $\sigma /(1-\sigma)$ where $\sigma$ is Poisson's ratio.

Because of (A.1),

$$
\int_{N} u_{\alpha} T_{\alpha \beta(1), \beta} d \xi^{1} d \xi^{2}=0,
$$

where, as usual, $N$ denotes a cross-section of the rod (on which $x^{0}=$ constant). Since $\boldsymbol{n}_{\beta(0)}$ have the values indicated in (7.24), by Green's theorem (A.18) can be written in the form 
(A.19) $\quad \oint_{N} u_{\alpha} T_{\alpha \beta(1)} n_{\beta(0)} d \tau-\int_{N} u_{\alpha, \beta} T_{\alpha \beta(1)} d \xi^{1} d \xi^{2}=0$

Because of the boundary condition (A.5) the first integral in (A.19) vanishes. Since $T_{\alpha \beta(1)}=T_{\beta \alpha(1)}$, the remaining integral can be written in the form

(A.20) $\quad \int_{N} \frac{1}{2}\left(u_{\alpha, \beta}+u_{\beta, \alpha}\right) T_{\alpha \beta(1)} d \xi^{1} d \xi^{2}$.

By substitution from (A.10), it is found that

(A.21) $\int_{N}\left[2\left(T_{12(1)}\right)^{2}+\frac{1}{2}\left(T_{11(1)}-T_{22(1)}\right)^{2}+\frac{1}{2}(1-2 \sigma)\left(T_{\alpha \alpha(1)}\right)^{2}\right] d \xi^{1} d \xi^{2}=0$.

Since $1-2 \sigma>0$, it is then necessary that

$$
T_{\alpha \beta(1)}=0
$$

Thus Saint-Venant's hypothesis has been proved for the finite displacement of thin rods.

UNiversity of Michigan, ANn Arbor, Mich. 